\title{
Transmission of a Detonation Across a Density Interface
}

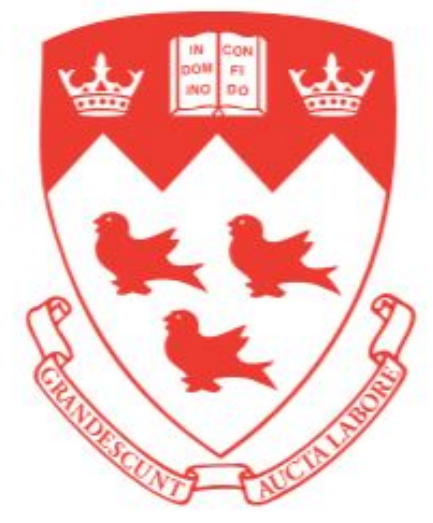

\section{Kelsey Tang Yuk}

Department of Mechanical Engineering McGill University

December 14, 2017

A thesis submitted to McGill University in partial fulfillment of the requirements for the degree of Master of Engineering 


\section{Abstract}

The present study investigates the transmission of a CJ detonation wave across a density interface. Associated with the transmitted detonation there is a reflected shock or expansion wave and a contact surface that separates the gases on each side of the contact surface initially. The problem is first studied theoretically. It is found that if there is a density decrease there is a transmitted strong detonation wave and a reflected expansion wave. If there is a density increase there is a transmitted CJ detonation wave with an expansion wave behind it and a reflected shock wave. Numerical simulations are then performed using two chemical kinetic models. They contain a transient process that occurs subsequent to the interaction due to a finite reaction zone thickness. After the transient relaxation process, the waves asymptotically approach their final state in the new medium. These final states are compared to the theoretical solution and are found to agree well qualitatively and quantitatively. 


\section{Résumé}

Ce travail étudie la transmission d'une onde de détonation CJ au travers une surface qui délimite deux régions de densités différentes. Associé à la détonation qui est transmise, il y a une onde de choc ou d'expansion qui est réfléchie, ainsi qu'une surface de contact qui sépare les deux gaz. Le problème est abordé initialement de manière analytique. On constate que, si la détonation passe vers un milieu à densité moins élevée, une forte détonation est transmise et une onde d'expansion est réfléchie. Si au contraire, la détonation passe vers un milieu à densité plus élevé, une détonation CJ avec une onde d'expansion derrière elle sont transmise, et une onde de choc est réfléchie. Le problème est ensuite abordé en effectuant des simulations numériques qui prennent en compte de l'épaisseur de la zone de réaction de la détonation. Elles incluent un processus transitoire qui se produit suite à l'intéraction de la détonation avec la surface qui délimite les deux régions de densités. Après ce processus de relaxation transitoire, les ondes s'approchent asymptotiquement de leur état final dans le nouveau milieu. Les états finaux obtenus ont été comparés à la solution analytique. Ils se sont avérés en accord qualitativement et quantitativement. 


\section{Acknowledgment}

Nothing happens in a vacuum, and the knowledge and wisdom I have gained would not have been possible without many brilliant people. I am forever grateful to my supervisor, Dr. John Lee, for accepting me as a graduate student on short notice. My growth is attributed to his patience in discussing fundamentals with me, his passion for the subject and his many tidbits of life wisdom. Secondly, my unofficial co-supervisor, Dr. Hoi Dick Ng. Without his guidance and his numerical integration code, I would have spent another year working. His generosity in helping me, someone he had no responsibility towards, is unprecedented. I also have to thank my colleague and friend XiaoCheng Mi. His help with some plots presented and his insights are greatly appreciated. Finally, I must thank all my family and friends, for providing that work/life balance, but more importantly for supporting me. In particular my parents, without whom I would not have had this opportunity, my sisters and my friends. 


\section{Contents}

$\begin{array}{ll}\text { List of Figures } & 7\end{array}$

$\begin{array}{ll}\text { List of Symbols } & 8\end{array}$

1 Introduction $\quad 10$

1.1 Non-uniformity of detonation gases . . . . . . . . . . . . . . . 10

1.2 Detonation theory . . . . . . . . . . . . . . . . . . . 12

2 Theoretical Analysis 13

2.1 Possible wave solutions . . . . . . . . . . . . . . . . . . 13

2.2 Governing equations . . . . . . . . . . . . . . . . . . 14

2.2.1 Example wave pattern . . . . . . . . . . . . . 16

2.3 Theoretical solution . . . . . . . . . . . . . . . . . . . . . 19

3 Numerical Analysis $\quad 21$

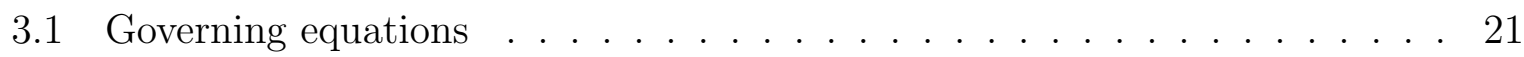

3.1.1 Single step Arrhenius kinetic model . . . . . . . . . . . . . . . . . 22

3.1.2 Two step induction-reaction kinetic model . . . . . . . . . . . 23

3.2 Numerical specifications . . . . . . . . . . . . . . . . . . 24

4 Relaxation Process $\quad 26$

4.1 Transient dynamics . . . . . . . . . . . . . . . . . . 26

4.1 .1 Density decrease ................... 26 
4.1 .2 Density increase . . . . . . . . . . . . . . . . . 32

4.1.3 Inert downstream medium . . . . . . . . . . . . . . . 38

4.2 Effect of $\Delta \rho \ldots \ldots \ldots \ldots \ldots$

4.2 .1 Density decrease ...................... 40

4.2.2 Density increase . . . . . . . . . . . . . . . . . 41

4.3 Effect of kinetic model . . . . . . . . . . . . . . . . . . . . . . . . . 42

4.3.1 Density decrease . . . . . . . . . . . . . . . . 43

4.3 .2 Density increase . . . . . . . . . . . . . . . . . 44

5 Theoretical and Numerical Comparison $\quad 46$

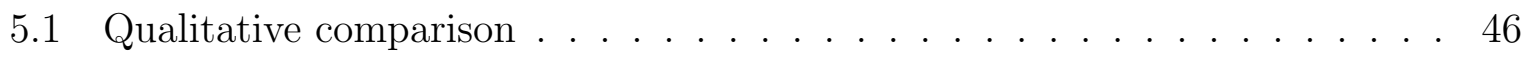

5.1 Density decrease ...................... 47

5.1 .2 Density increase . . . . . . . . . . . . . . . . 48

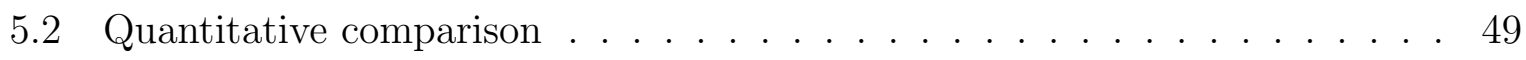

6 Conclusion $\quad 51$

$\begin{array}{ll}\text { Appendix A } & 52\end{array}$

$\begin{array}{ll}\text { Appendix B } & 58\end{array}$

$\begin{array}{ll}\text { References } & 61\end{array}$ 


\section{List of Figures}

1.1 Detonation wave transmission across an interface . . . . . . . . . . 10

2.1 Schematic for CJ detonation transmission across an interface . . . . . . . . 14

2.2 A transmitted strong or CJ detonation with a reflected shock wave . . . . 17

2.3 Theoretical solution . . . . . . . . . . . . . . . . . . 19

2.4 Degree of overdrive of transmitted detonation for various incident detontation Mach numbers and density changes . . . . . . . . . . . . . 20

3.1 Single step Arrhenius model ZND profile for $E_{a}=20, Q=50, \gamma=1.2$. . 22

3.2 Two step induction-reaction model ZND profile for $k r=0.887, \epsilon_{i}=8, \epsilon_{r}$ $=1, Q=50, \gamma=1.2 \ldots \ldots \ldots \ldots \ldots$

4.1 Relaxation process for $\Delta \rho=-25 \%$ with single step Arrhenius kinetics with $E_{a}=20, Q=50$ and $\gamma=1.2 \ldots \ldots \ldots$. . . . . . . . . . 27

4.2 Pressure profiles and schlieren plot for $\Delta \rho=-25 \%$ and single step Arrhenius kinetics with $E_{a}=20, Q=50, \gamma=1.2 \ldots \ldots$. . . . . . . . 28

4.3 Temperature profiles and schlieren plot for $\Delta \rho=-25 \%$ and single step

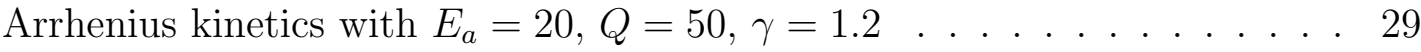

4.4 Reaction rate, $\dot{\omega}$ and Schlieren plot for $\Delta \rho=-25 \%$ and single step Arrhenius kinetics with $E_{a}=20, Q=50, \gamma=1.2 \ldots \ldots$. . . . . . . . 31

4.5 Relaxation process for $\Delta \rho=20 \%$ with single step Arrhenius kinetics with $E_{a}=20, Q=50$ and $\gamma=1.2 \ldots \ldots \ldots$. . . . . . . . . . . . . . . .

4.6 Pressure profiles and Schlieren plot for $\Delta \rho=20 \%$ and single step Arrhenius

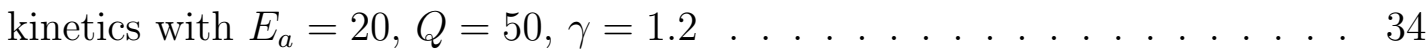

4.7 Temperature profiles and Schlieren plot for $\Delta \rho=20 \%$ and single step Arrhenius kinetics with $E_{a}=20, Q=50, \gamma=1.2 \ldots \ldots . \ldots . . \ldots 35$

4.8 Reaction rate, $\dot{\omega}$ and Schlieren plot for $\Delta \rho=20 \%$ and single step Arrhenius kinetics with $E_{a}=20, Q=50, \gamma=1.2 \ldots \ldots$. . . . . . . . 37

4.9 Pressure and Schlieren plot for single step Arrhenius kinetics with $E_{a}=20$, $Q=50, \gamma=1.2$ to an inert gas where $\Delta \rho=-25 \%$. . . . . . . . . 38

4.10 Pressure and Schlieren plot for single step Arrhenius kinetics with $E_{a}=20$, $Q=50, \gamma=1.2$ to an inert gas where $\Delta \rho=20 \%$. . . . . . . . . 39

4.11 Relaxation process for single step Arrhenius kinetics with $E_{a}=20, Q=50$, $\gamma=1.2$ and $\Delta \rho=-15 \%,-25 \%,-35 \% \ldots \ldots \ldots$. . . . . . . 41 
4.12 Relaxation process for single step Arrhenius kinetics with $E_{a}=20, Q=50$, $\gamma=1.2$ and $\Delta \rho=+10 \%,+20 \%,+30 \% \ldots \ldots \ldots \ldots \ldots \ldots$

4.13 Relaxation process for the two step kinetic model with $\epsilon_{i}=8, \epsilon_{r}=1$, $k_{i}=0.779, k_{r}=0.887, Q=50, \gamma=1.2$ and $\Delta \rho=-25 \% \ldots \ldots \ldots$

4.14 Relaxation process for the two step kinetic model with $\epsilon_{i}=8, \epsilon_{r}=1$, $k_{i}=0.779, k_{r}=0.887, Q=50, \gamma=1.2$ and $\Delta \rho=+20 \% \ldots \ldots 44$

5.1 Transmitted detonation for density decrease $\Delta \rho=-25 \% \ldots \ldots$. . . . 47

5.2 Transmitted detonation for a density increase with $E_{a}=20, Q=50$, $\Delta \rho=+20 \%$ and $\gamma=1.2 \ldots \ldots \ldots \ldots \ldots \ldots$

5.3 Degree of overdrive, $f$ of the transmitted detonation for $\Delta \rho=-35 \%$ to $30 \%$. Comparison of the two kinetic models and the theoretical analysis . . . . 49

A.1 Case 1: A transmitted strong or CJ detonation and a reflected shock wave 52

A.2 Case 2: A transmitted CJ detonation with expansion wave and a reflected shock wave . . . . . . . . . . . . . . . . . . . 53

A.3 Case 3: A transmitted strong or CJ detonation and a reflected expansion

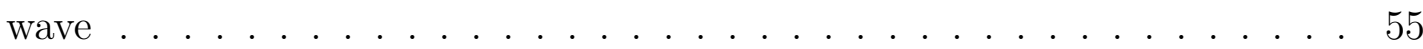

A.4 Case 4: A transmitted CJ detonation with an expansion wave and a reflected expansion wave . . . . . . . . . . . . . . 56

B.1 Transmission of a shock wave across an interface . . . . . . . . . 58 


\section{List of Symbols}

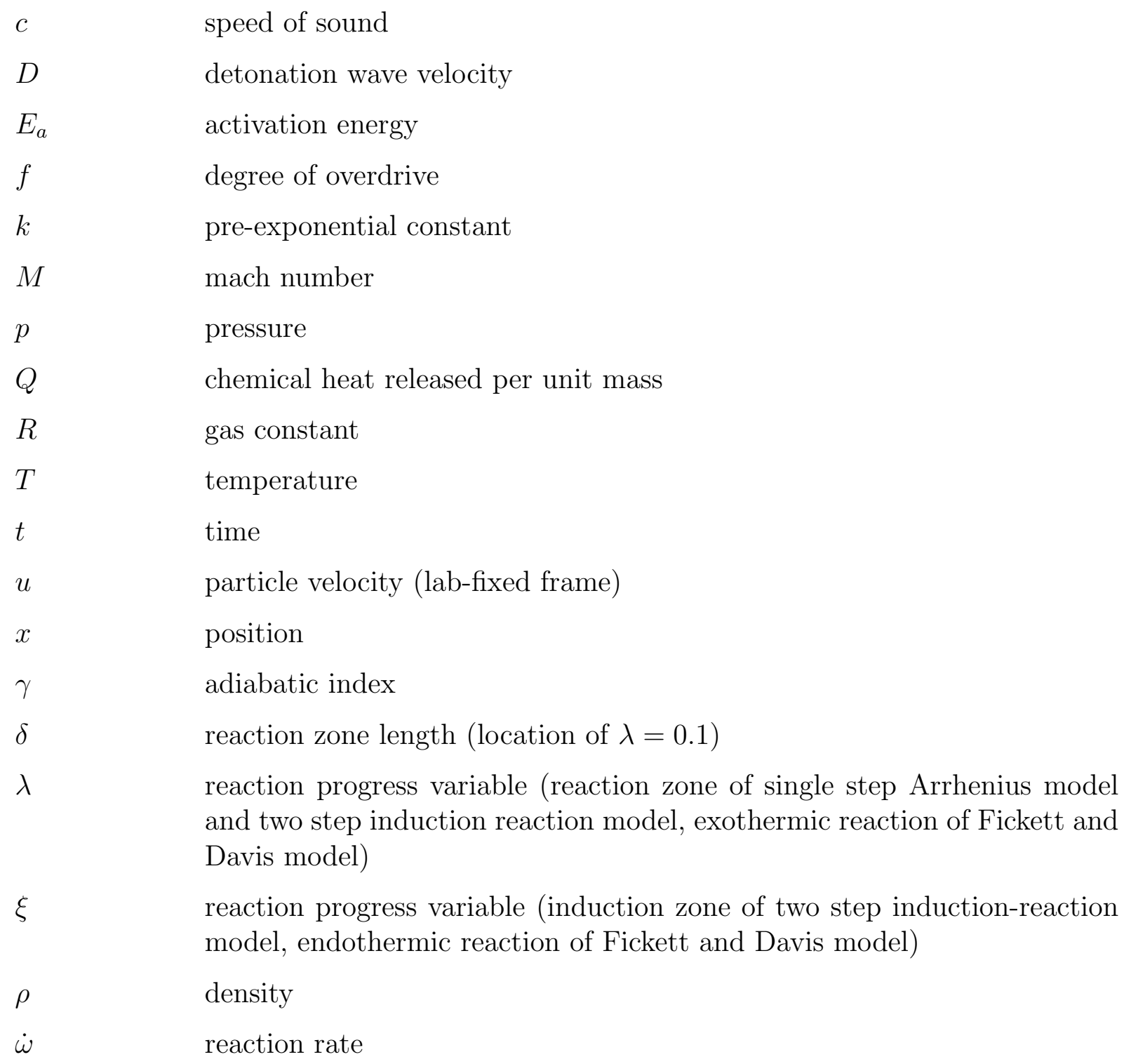




\section{Subscripts}

$+\quad$ property behind the shock/expansion wave (for a detonation corresponds to behind the leading shock wave)

- $\quad$ property in front of the shock/expansion wave (for a detonation corresponds to in front of the leading shock wave)

$0 \quad$ property in the initial unburnt gas

$D \quad$ property of a detonation wave

$d \quad$ property at the exit of the detonation wave

$i \quad$ property of the incident wave

$r \quad$ property of the reflected wave

$s \quad$ property of a shock wave

$t \quad$ property of the transmitted wave

\section{Abbreviations}

CJ Chapman-Jouget

CV Control volume

MUSCL monotonic upwind scheme for conservation laws

SLIC slope-limited centered

VN von Neumann

WAF weighted average flux

ZND Zel'dovich von Neumann Döring 


\section{Chapter 1}

\section{Introduction}

\subsection{Non-uniformity of detonation gases}

Inhomogeneities are inevitable in practical explosive mixtures. For example, in the accidental release of combustion fuel into the atmosphere, composition non-uniformities are common in the gaseous fuel-air cloud. Temperature, and hence density gradients may be formed due to temperature differences between the fuel released, ground or other structures and the ambient atmosphere.

The response of a shock wave to inhomogeneities is almost instantaneous, since it has negligible thickness compared to the scale of non-uniformities present. However, the response of a detonation to perturbations due to inhomogeneities may involve length and

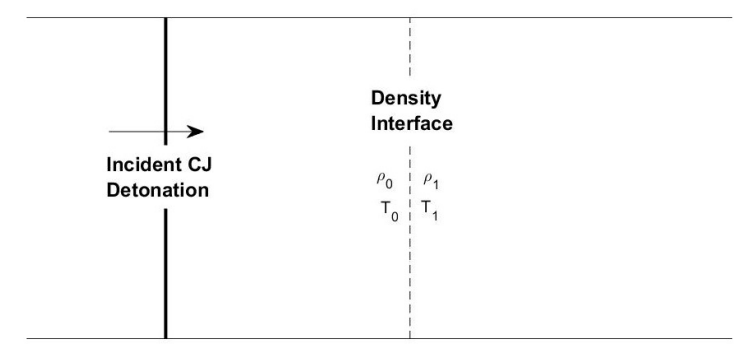

Figure 1.1: Detonation wave transmission across an interface 
time scales in excess of the intrinsic cellular instability of the detonation front. Thus, the propagation will be unsteady over scales much larger than that of the cellular instability.

The propagation of detonation waves in non-uniform mixtures has not been extensively studied. Early studies include those of Strehlow et al. [1], Bjerketvedt et al. [2] and Thomas et al. [3] on the transmission of a detonation from a reactive to inert medium. Other more recent studies have followed on this reactive to inert interaction problem $[4,5]$. However, transmission to a different reactive mixture has also recently been gathering interest. Kuznetsov et al. $[6,7]$ have looked at the phenomenon of detonation transmission to a mixture of lower sensitivity. Li et al. [8] studied experimentally the transmission from propane-oxygen to propane-air mixtures.

However, experimentally it is difficult to create reproducible, controlled inhomogeneities. Recently, in an attempt to create a well defined discontinuity, the head on collision of a detonation wave with a weak shock wave was studied $[9,10]$. Thus the detonation wave was subjected to a velocity, pressure, temperature as well as density jump. The present study looks at the simpler case of the detonation wave subject to a change in density and temperature numerically as shown in figure 1.1. To focus on the mechanism of the interaction, an abrupt interface is considered rather than a density gradient that introduces further complexities.

In the first part of the study a standard wave interaction analysis is carried out to establish a theoretical solution. Then numerical simulations are performed using two kinetic models. The transient relaxation resulting from the interaction is discussed. Finally, the downstream results of the numerical simulation are compared to the theoretical solution. 


\subsection{Detonation theory}

In one-dimension, a detonation wave is classically pictured to begin with a leading shock wave that adiabatically compresses and heats the reactants, allowing ignition. This shock wave is coupled with a reaction zone where combustion occurs. The energy released in the combustion process and expansion of gases in the reaction zone provide the thrust that directly supports the shock wave [11].

A control volume (CV) analysis of the detonation wave, essentially neglecting its structure, considers only equilibrium thermodynamics. There is a range of velocities of the wave that satisfies equilibrium. This begins at a unique, minimum velocity solution for which the product gases are at local sonic speed. This was postulated by Chapman and Jouguet (CJ criterion) to be the one physically realized and thus is called the CJ detonation velocity, $D_{C J}$. For velocities higher than $D_{C J}$ there exist two possible solutions

for the product state. For one the local product flow is supersonic, called the weak solution and for the other subsonic, called the strong solution. However, for this CV analysis, the weak detonation solution can be eliminated in general if ignition by adiabatic shock compression is assumed $[11,12]$.

If the detonation structure is considered, the steady, one-dimensional flow field for the CJ criterion was solved by Zeldovich [13], von Neumann [14], and Döring [15], known as the ZND solution. Following the shock compression, there is a region of relatively constant properties (slow reaction), called the induction zone, followed by rapid reaction. 


\section{Chapter 2}

\section{Theoretical Analysis}

If the detonation is assumed to be a discontinuity, then a standard wave interaction analysis of the transmission problem can be carried out.

\subsection{Possible wave solutions}

The $x$ - $t$ diagram of the wave process is shown in figure 2.1. The flow behind the incident detonation is assumed to be uniform. Across the interface, a transmitted detonation wave is obtained. A reflected wave and a contact surface will also be formed. The contact surface separates the gas initially on each side of the density discontinuity.

The problem is defined for a given incident detonation Mach number, $M_{D, i}=M_{C J, i}$ and properties at each side of the interface, whether there is a density increase or decrease. The transmitted detonation can be a strong, weak or CJ detonation wave. However, as mentioned in section 1.2, the weak detonation solution can be eliminated in general.

If the transmitted detonation wave is strong, the product flow is subsonic and uniform up to the contact surface. If the transmitted detonation wave is CJ, then the flow can

either be uniform up until the contact surface or there can be an expansion wave trailing behind the transmitted detonation wave [9]. Thus, there can be either a transmitted 


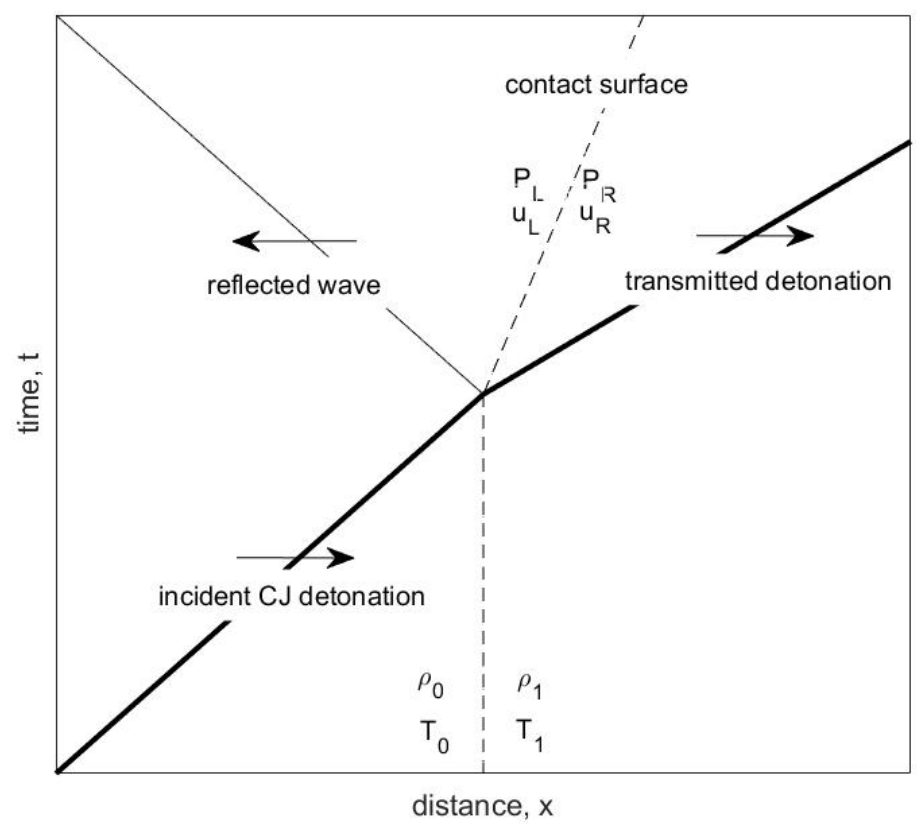

Figure 2.1: Schematic for CJ detonation transmission across an interface

strong or CJ detonation wave with uniform flow behind it or a transmitted CJ detonation wave with an expansion wave behind it. Depending on the boundary conditions, the reflected wave can be either a shock wave or an expansion wave.

\subsection{Governing equations}

The governing equations presented apply under the assumption of one-dimensional, steady flow of an ideal gas with constant specific heats.

The boundary conditions to be satisfied across the contact surface will be constant pressure, $p$ and particle velocity, $u\left(u_{L}=u_{R}\right.$ and $\left.p_{L}=p_{R}\right)$. For the incident and transmitted detonation waves, the relationships across the detonation wave are given by the relations named after Rankine [16] and Hugoniot [17]: 


$$
\begin{aligned}
& \frac{p_{D+}}{p_{-}}=\frac{\gamma+\eta_{D} \pm \gamma s}{\eta_{D}(1+\gamma)} \\
& \frac{\rho_{D+}}{\rho_{-}}=\frac{\gamma+1}{\left(\gamma+\eta_{D} \mp s\right)}=\frac{D}{D-u_{D+}} \\
& M_{D}=\frac{D}{c_{-}} \\
& \text {with } \\
& s=\sqrt{\left(1-\eta_{D}\right)^{2}-K \eta_{D}} \quad \text { with } \\
& \eta_{D}=\frac{1}{M_{D}{ }^{2}}=\left(1+\frac{K}{2}\right)-\sqrt{K\left(1+\frac{K}{4}\right)} \text { with } \\
& K=2\left(\gamma^{2}-1\right) \frac{Q}{\gamma p_{-} v_{-}}
\end{aligned}
$$

$p, \rho, v$ and $u$ are pressure, density, specific volume and particle velocity respectively. $c$ is the speed of sound. $M_{D}$ is the Mach number of the detonation wave, $D$ is its velocity and $Q$ is the chemical heat released per unit mass in the reaction. The adiabatic index, $\gamma$ is assumed to be the same for the reactants and products. In the above equations, the "+" and "-" indicate the properties behind and in front of the wave, respectively. The " $D$ " subscript indicates that these flow values are the product flow at the end of the detonation reaction zone. In this way we differentiate between, for example, $p_{D+}$ at the exit of the wave and $p_{+}$behind the leading shock front.

The two possible solutions representing the strong and weak detonation solutions are represented by the two signs in front of "s". The top denotes the strong and the bottom sign the weak solution.

For $s=0$ the CJ solution is obtained and the flow is sonic behind the wave. If there is an expansion wave behind the CJ detonation, the Riemann invariants are constant along a C- characteristic [18] and the isentropic relationships apply, i.e.: 


$$
\begin{aligned}
& u_{+}-\frac{2}{\gamma-1} c_{+}=u_{-}-\frac{2}{\gamma-1} c_{-} \\
& \frac{c_{+}}{c_{-}}=\left(\frac{p_{+}}{p_{-}}\right)^{\frac{\gamma-1}{2 \gamma}}
\end{aligned}
$$

For the reflected shock wave, the Rankine-Hugoniot $[16,17]$ equations across the shock wave are:

$$
\begin{aligned}
& \frac{p_{+}}{p_{-}}=\frac{2 \gamma M_{s}^{2}-(\gamma-1)}{\gamma+1} \\
& \frac{u_{+}-u_{-}}{c_{-}}=\frac{2\left(M_{s}^{2}-1\right)}{(\gamma+1) M_{s}}
\end{aligned}
$$

Here $M_{s}$ is the shock wave Mach number and $c$ is the sound speed.

If the reflected wave is an expansion wave, the characteristic equations [18] and the isentropic relationships apply, i.e.:

$$
\begin{aligned}
& u_{+}+\frac{2}{\gamma-1} c_{+}=u_{-}+\frac{2}{\gamma-1} c_{-} \\
& \frac{c_{+}}{c_{-}}=\left(\frac{p_{+}}{p_{-}}\right)^{\frac{\gamma-1}{2 \gamma}}
\end{aligned}
$$

For a given incident CJ detonation and properties on each side of the interface, the flow properties between the incident detonation and the reflected wave can be found using the Rankine-Hugoniot relationships, equation 2.1, with $s=0$.

\subsubsection{Example wave pattern}

Consider the possibility of a transmitted strong (or CJ) detonation with uniform flow behind it and a reflected shock wave (figure 2.2). The properties at 0, 1 and 2 are 


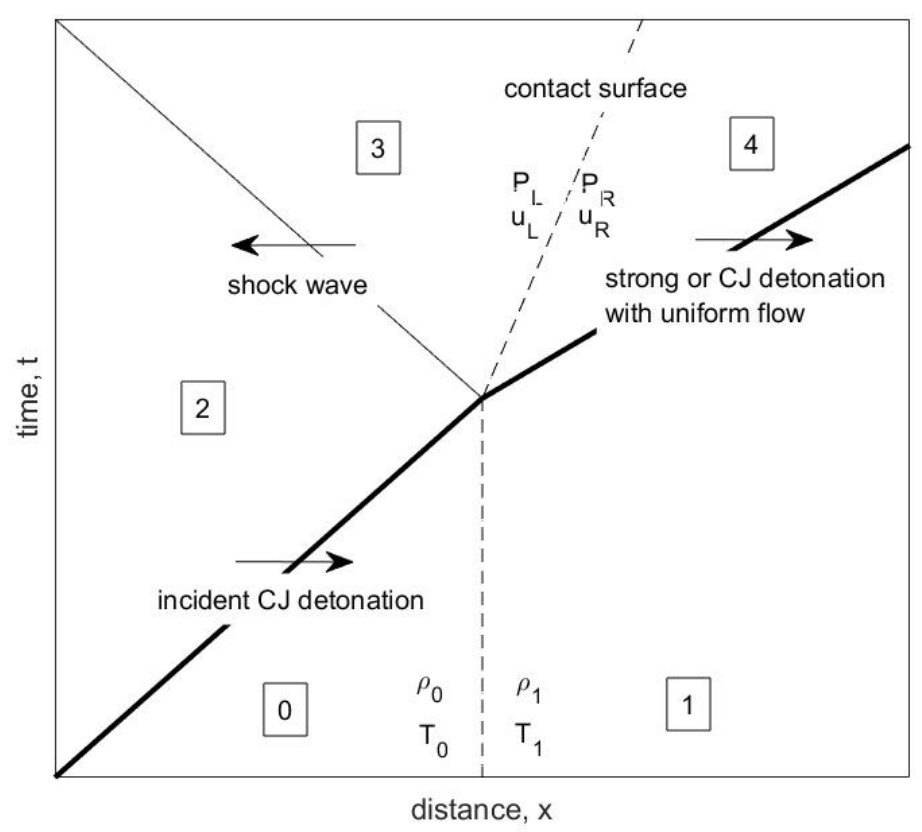

Figure 2.2: A transmitted strong or CJ detonation with a reflected shock wave

known. The pressure, $p$ and particle velocity, $u$ behind the transmitted detonation can be expressed in terms of the transmitted detonation strength, $\eta_{D, t}$ using equation 2.1:

$$
\begin{aligned}
& \frac{p_{4}}{p_{1}}=\frac{\eta_{D, t}+\gamma(1+s)}{\eta_{D, t}(1+\gamma)} \\
& u_{4}=D_{t}\left[1-\frac{\gamma+\eta_{D, t}-s}{\gamma+1}\right] \text { where } \\
& D_{t}=\frac{c_{1}}{\sqrt{\eta_{D, t}}} \\
& s=\sqrt{\left(1-\eta_{D, t}\right)^{2}-K \eta_{D, t}} \\
& K=2\left(\gamma^{2}-1\right) \frac{Q}{\gamma p_{1} v_{1}}
\end{aligned}
$$

Since there is uniform flow behind the wave, $p$ and $u$ on the right of the contact surface are given by: 


$$
\begin{aligned}
& p_{R}=p_{4} \\
& u_{R}=u_{4}
\end{aligned}
$$

Similarly, $p$ and $u$ behind the reflected shock wave at 3 can be expressed in terms of the reflected shock strength $M_{s, r}$, using the normal shock equation 2.3 and considering the fact that it is a left facing shock (particles entering from the left):

$$
\begin{aligned}
& \frac{p_{3}}{p_{2}}=\frac{2 \gamma M_{s, r}-(\gamma-1)}{\gamma+1} \\
& u_{3}=u_{2}-\left(p_{3}-p_{2}\right)\left[\frac{\frac{2}{(\gamma+1) \rho_{2}}}{p_{3}+\frac{\gamma-1}{\gamma+1} p_{2}}\right]^{\frac{1}{2}}
\end{aligned}
$$

Again these are equal to those on the left of the contact surface:

$$
\begin{aligned}
& p_{L}=p_{3} \\
& u_{L}=u_{3}
\end{aligned}
$$

Thus, we have $p_{L}, u_{L}$, and $p_{R}, u_{R}$ in terms of the strengths of the respective waves $\left(M_{s, r}\right.$ and $\left.\eta_{D, t}\right)$. By imposing $p_{\mathrm{R}}=p_{\mathrm{L}}$ and $u_{\mathrm{R}}=u_{\mathrm{L}}$ across the contact surface, we find an equation with only one unknown, $\eta_{D, t}$ :

$$
\begin{aligned}
& \frac{c_{1}}{\sqrt{\eta_{D, t}}}\left[1-\left(\frac{\gamma+\eta_{D, t}-s}{\gamma+1}\right)\right]-u_{2} \\
& =\left(p_{2}-\frac{\eta_{D, t}+\gamma(1+s)}{\eta_{D, t}(\gamma+1)}\right)\left(\frac{\frac{2}{(\gamma+1) \rho_{2}}}{\frac{\eta_{D, t}+\gamma(1+s)}{\eta_{D, t}(\gamma+1)}+\frac{\gamma-1}{\gamma+1} p_{2}}\right)^{\frac{1}{2}}
\end{aligned}
$$




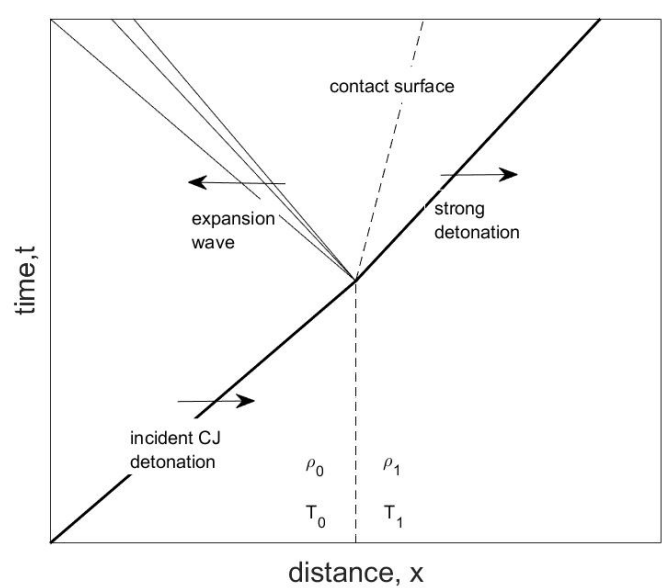

(a) Density decrease solution

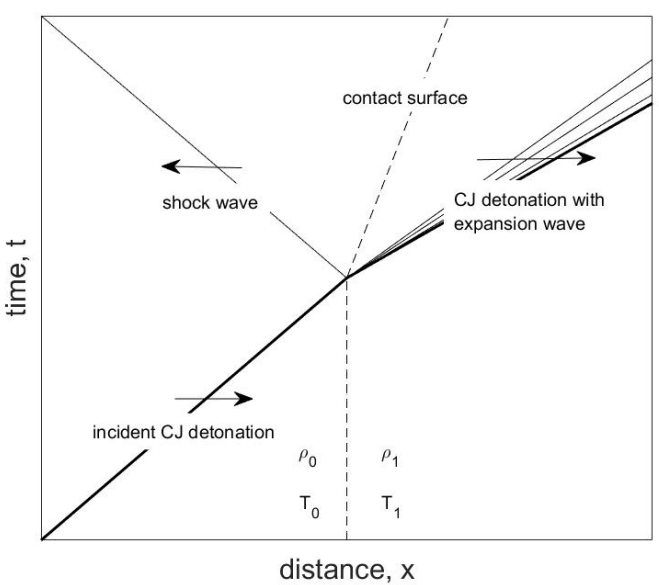

(b) Density increase solution

Figure 2.3: Theoretical solution

Equation 2.9 can then be solved iteratively for $\eta_{D, t}$ to determine if a solution exists.

In this way, for each combination of transmitted and reflected waves, combination we look for a flow field solution that satisfies mechanical equilibrium (i.e. equal pressure and particle velocity) at the contact surface. The derivation of equations for all other wave combinations are given in appendix A.

\subsection{Theoretical solution}

The problem was solved for a range of incident detonation Mach numbers $(\sim 3.0$ to $\sim 7.3)$ with a range of density changes $(-50 \%$ to $+50 \%)$.

The solution wave pattern was found to depend on whether there is a density increase or decrease. For a density decrease (and temperature increase), a transmitted strong detonation and a reflected expansion wave (figure 2.3a) are obtained. For a density increase (and temperature decrease), a transmitted CJ detonation with an expansion wave and a reflected shock wave (figure $2.3 \mathrm{~b}$ ) are obtained.

The degree of overdrive of the transmitted detonation is defined as $f=\frac{D_{t}^{2}}{D_{C J}^{2}}$ where $D_{t}$ 


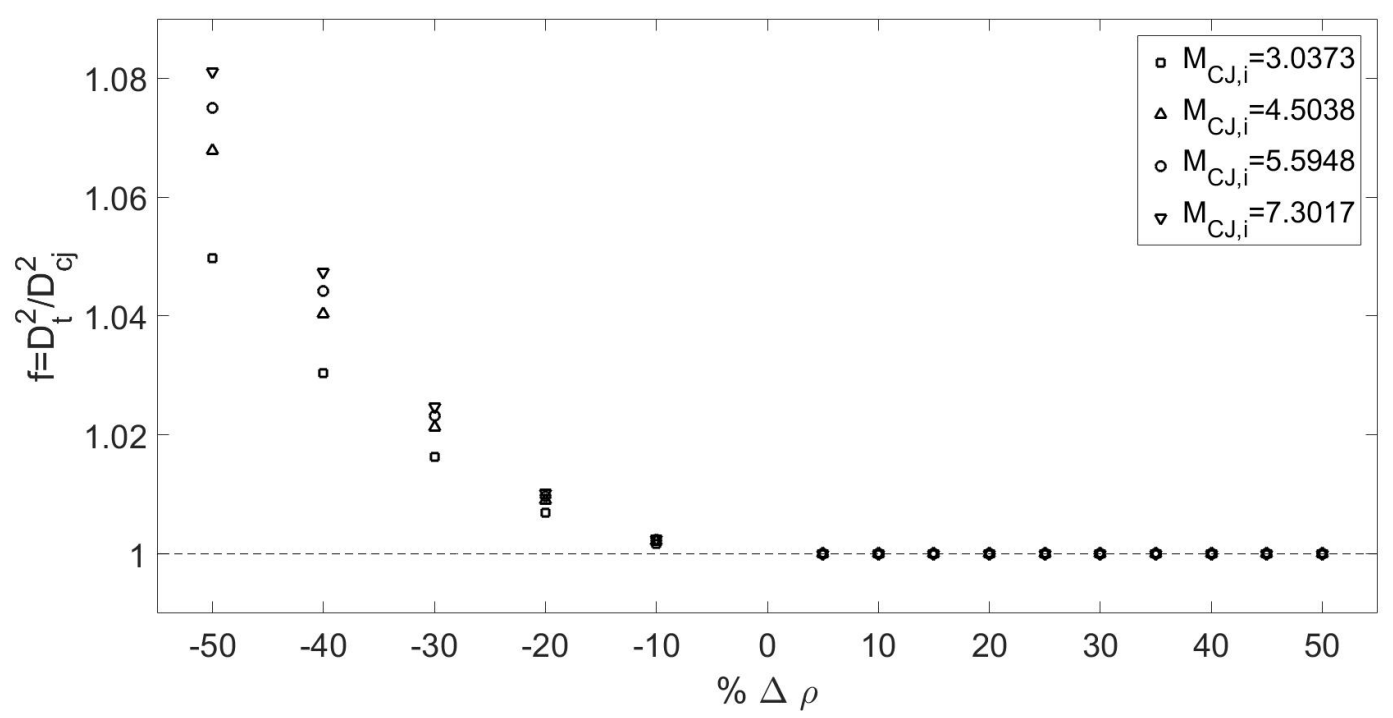

Figure 2.4: Degree of overdrive of transmitted detonation for various incident detontation Mach numbers and density changes

is the speed of the transmitted detonation and $D_{C J}$ the CJ detonation in that medium (on the right of the interface). This is plotted against the percentage density change for selected incident detonation Mach numbers, $M_{D, i}=M_{C J, i}$ in figure 2.4. For all cases, the transmitted detonation is strong $(f>1)$ for a density decrease and CJ $(f=1)$ for a density increase. The degree of overdrive, $f$, increases with increasing magnitude of density decrease, $|\Delta \rho|$ and increasing $M_{C J, i}$. 


\section{Chapter 3}

\section{Numerical Analysis}

If we consider the detonation to have a finite reaction zone thickness, the transient, unsteady response of the detonation must be taken into account. Thus, the problem must be solved numerically.

\subsection{Governing equations}

Again the governing equations assume one-dimensional flow of an ideal gas with constant specific heats. They are the set of one-dimensional, unsteady, reactive Euler equations given by:

$$
\frac{\partial \mathbf{U}}{\partial t}+\frac{\partial \mathbf{F}(\mathbf{U})}{\partial x}=\mathbf{S}(\mathbf{U})
$$

where,

$$
\mathbf{U}=\left[\begin{array}{c}
\rho \\
\rho u \\
\rho e \\
\rho \lambda
\end{array}\right], \mathbf{F}(\mathbf{U})=\left[\begin{array}{c}
\rho u \\
\rho u^{2}+p \\
(\rho e+p) u \\
\rho u \lambda
\end{array}\right], \mathbf{S}(\mathbf{U})=\left[\begin{array}{c}
0 \\
0 \\
0 \\
\dot{\omega}
\end{array}\right]
$$




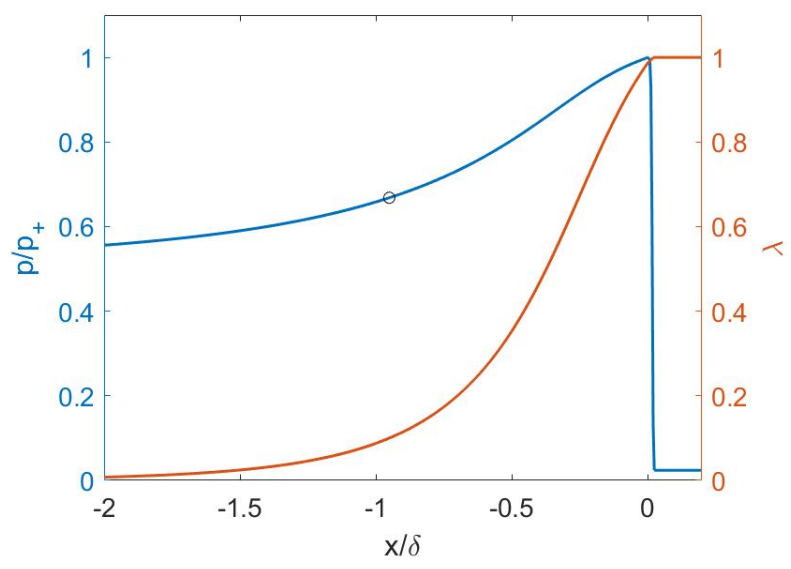

Figure 3.1: Single step Arrhenius model ZND profile for $E_{a}=20, Q=50, \gamma=1.2$

Here $e$ is the total energy per unit mass:

$$
e=\frac{p}{(\gamma-1) \rho}+\frac{u^{2}}{2}+\lambda Q
$$

The governing equations are coupled with a chemical kinetic law for the reaction rate, $\dot{\omega}$. The reaction progress variable is denoted as $\lambda$, where $\lambda=1$ for reactants and $\lambda=0$ for products. In this study, two different reaction rate laws were considered, i.e. the single step Arrhenius reaction law and two step induction-reaction law.

\subsubsection{Single step Arrhenius kinetic model}

The simplest case is a single step law in the Arrhenius [19] form given by:

$$
\dot{\omega}=-k \rho \lambda \exp \left(\frac{-E_{a}}{T}\right)
$$

where $E_{a}$ is the activation energy, $T$ is the temperature and $k$ is a pre-exponential constant for a given mixture that is used to define spatial and temporal scales.

The ZND detonation wave structure with this kinetic law is shown in figure 3.1 for $E_{a}$ $=20, Q=50$ and $\gamma=1.2$. The blue line shows the pressure, $p$ normalized by the von 
Neumann shock pressure, $p_{+}$and the orange line is the reaction progress variable $\lambda$. The horizontal axis is distance, $x$ normalized by the reaction zone length, $\delta$. Here $\delta$ is defined as the location at which $\lambda=0.1$ or equivalently where $90 \%$ of heat has been released. The axis is shifted such that $x / \delta=0$ corresponds to the detonation wave front and the small circle on the pressure plot indicates the reaction zone end, $\delta=-1$ and $\lambda=0.1$. At the shock front $(x / \delta=0)$ there is an abrupt increase in pressure. Pressure decreases as the reaction progress variable approaches 0 through the reaction zone.

\subsubsection{Two step induction-reaction kinetic model}

A more realistic law accounts for an induction zone before the reaction zone. The reaction zone is modeled in the same way and the induction zone is also of an Arrhenius type. This model has previously been used by Short and Sharpe in [20]. It is given by:

$$
\begin{aligned}
& \frac{d(\rho \lambda)}{d t}=-(1-H(1-\xi)) k_{R} \rho \lambda \exp \left(\frac{-E_{R}}{T}\right) \\
& \frac{d(\rho \xi)}{d t}=-H(1-\xi) \rho k_{I} \lambda \exp \left[E_{I}\left(\frac{1}{T_{+}}-\frac{1}{T}\right)\right] \\
& \text { where, } \\
& H(1-\xi)= \begin{cases}1 & \xi>0 \\
0 & \xi \leq 0\end{cases}
\end{aligned}
$$

There is a reaction zone progress variable, $\lambda$ and an induction zone progress variable, $\xi$. They decrease from 1 at the beginning of their respective zones to 0 at the end. There are also two activation energies and pre-exponential constants, $E_{I}$ and $k_{I}$ for the induction zone and $E_{R}$ and $k_{R}$ for the reaction zone. Parameters are adjusted to vary the relative length of the induction and reaction zones. $T_{+}$is the temperature at the shocked state. 


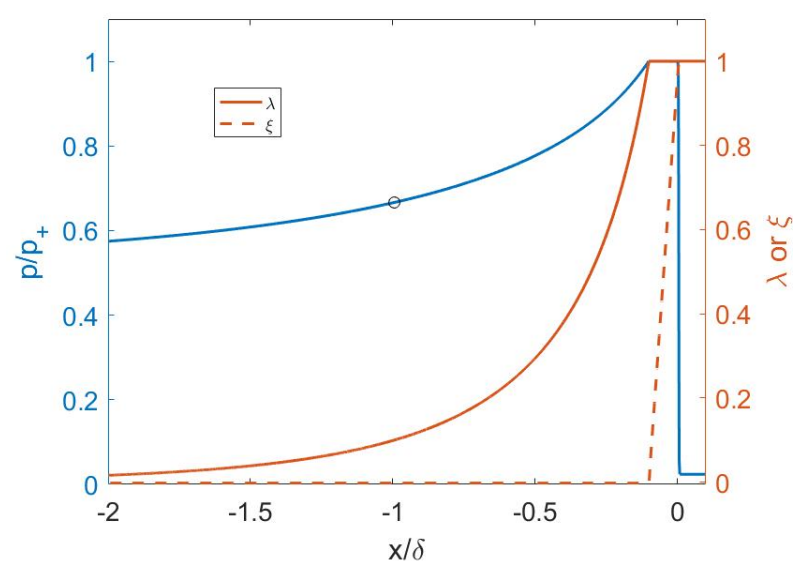

Figure 3.2: Two step induction-reaction model ZND profile for $k r=0.887, \epsilon_{i}=8, \epsilon_{r}=$ $1, Q=50, \gamma=1.2$

The parameters $\epsilon_{i}$ and $\epsilon_{r}$ are defined to be $E_{i} / \mu$ and $E_{r} / \mu$, respectively, where $\mu$ is the temperature jump across the leading shock of the detonation, i.e.:

$$
\mu=\frac{T_{+}}{T_{-}}=\frac{\left[2 \gamma M_{D}^{2}-(\gamma-1)\right]\left[(\gamma-1) M_{D}^{2}+2\right]}{(\gamma+1)^{2} M_{D}^{2}}
$$

Figure 3.2 shows the detonation profile for this reaction law with $k r=0.887, k i=$ $0.779, \epsilon_{i}=8, \epsilon_{r}=1, Q=50$ and $\gamma=1.2$. The reaction zone is the same as before, $\lambda$ (solid orange line) decreases from 1 to 0 as pressure, $\frac{p}{p_{+}}$decreases. After the shock front, $\xi$ (dashed orange line) decreases from 1 to 0 at the end of the induction zone and beginning of the reaction zone. The small circle on the pressure plot indicates the location of $\lambda=0.1$ which is defined as the end of the reaction zone. The horizontal axis $x / \delta$ is similarly scaled and shifted such that $x / \delta=0$ corresponds to the leading shock front.

\section{$3.2 \quad$ Numerical specifications}

The governing equations are numerically integrated using the method of fractional steps and two second-order finite difference schemes. These are the slope-limited centered 
(SLIC) and the upwind weighted averaged flux (WAF) schemes. For more information on these schemes see [21].

The upwind WAF scheme with an exact Riemann solver was used with the single step Arrhenius kinetic model. The SLIC scheme was used with the two step induction-reaction kinetic model. These codes have been used in gaseous detonation simulations in the past $[22,23]$ and therefore have been verified.

The computational domain was defined as a one-dimensional Cartesian grid. In the simulation, a rightward-propagating detonation was initialized as the ZND wave solution near the end of the domain. Transmissive boundary conditions were applied on the left and right boundaries of this domain.

The resolution was defined using a characteristic length scale for each reaction law. For the single step Arrhenius kinetic model, the characteristic length scale corresponds to the half reaction zone length of the initial steady ZND wave. For the two step inductionreaction kinetic model, the induction zone length is used as the characteristic length scale. A resolution of 100 grid points per characteristic length was used as verified for convergence in $[22,23]$.

To achieve this the appropriate pre-exponential constants were scaled such that the characteristic length scales were unity. For the single step Arrhenius kinetic model, this was the pre-exponential constant $k$. For the two step induction-reaction kinetic model, $k_{i}$ is set to be the particle velocity behind the shock front in the shock-fixed frame, $u_{D,+}$.

All quantities were made dimensionless with respect to the initial unburnt gas state. The detonation was allowed to propagate over a sufficient distance to reach its steady state before the interface was encountered (at minimum a distance of 400 characteristic reaction zone lengths of the incident ZND detonation). 


\section{Chapter 4}

\section{Relaxation Process}

Since the detonation has a reaction zone of finite thickness, the final state will not be achieved instantaneously downstream of the density interface. There will be an unsteady period during which the detonation responds to the change in density. Subsequently, the detonation will asymptotically approach its final state downstream.

\subsection{Transient dynamics}

The transient dynamics of the relaxation process is first studied using the simpler kinetic model, single step Arrhenius kinetics.

\subsubsection{Density decrease}

A density decrease and temperature increase across the interface are first considered. Figure 4.1 shows the variation of the leading shock pressure, $p_{+}$with distance, $\frac{x}{\delta}$. Pressure is normalized by the initial pressure of the unburnt material, $p_{0}$ and distance is normalized with the reaction zone length, $\delta$. The $x$-axis is shifted such that the interface is located at $\frac{x}{\delta}=0$. A density decrease of $\Delta \rho=-25 \%$ and the single step Arrhenius model are 


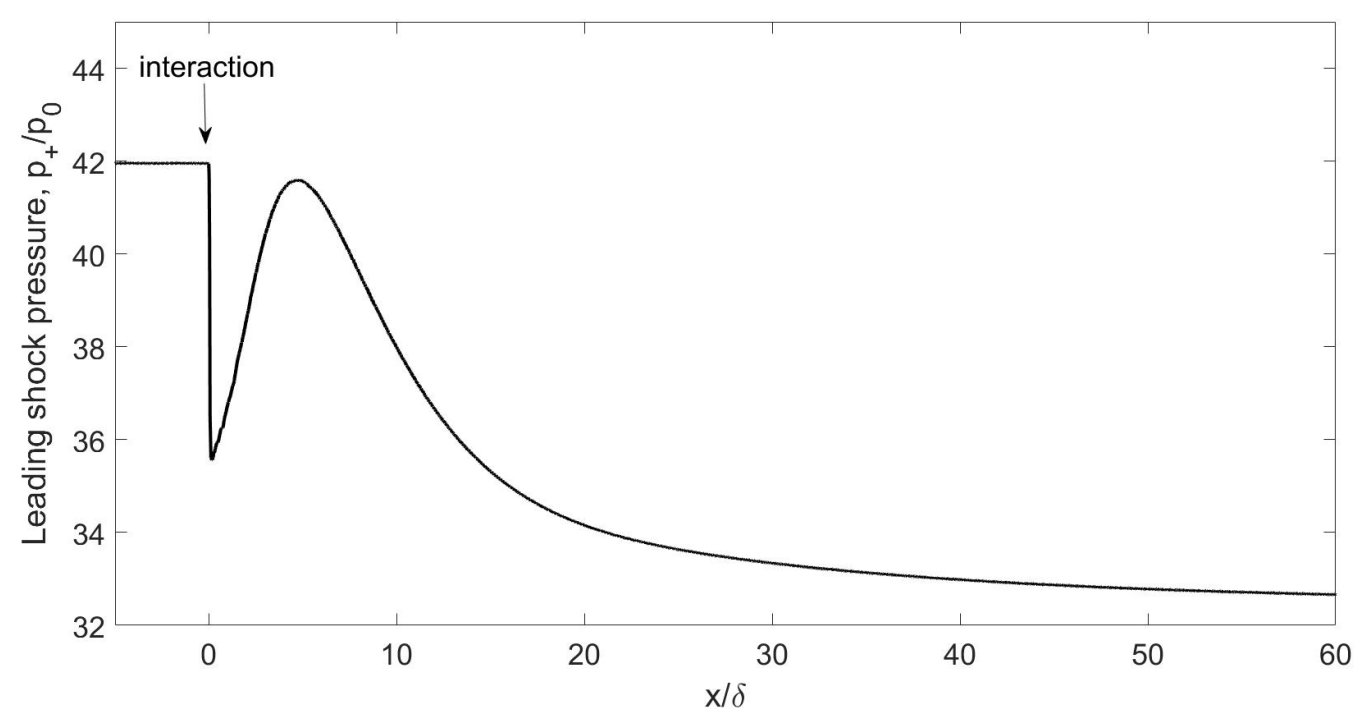

Figure 4.1: Relaxation process for $\Delta \rho=-25 \%$ with single step Arrhenius kinetics with $E_{a}=20, Q=50$ and $\gamma=1.2$

used with $E_{a}=20, Q=50$ and $\gamma=1.2$.

Immediately downstream of the interface the shock pressure ratio, $\frac{p_{+}}{p_{0}}=\frac{p_{+}}{p_{-}}$undergoes an abrupt decrease to about $80 \%$ its initial value. This is followed by a rapid reacceleration to a shock pressure ratio $\frac{p_{+}}{p_{-}} \sim 41.6$, at a distance of $\frac{x}{\delta} \sim 4.8$ (or 4.8 reaction zone lengths, $\delta$ downstream of the interface). Subsequently, the shock decays asymptotically to the final steady state, at pressure ratio $\frac{p_{+}}{p_{-}} \sim 32.5$. This relaxation length is $\frac{x}{\delta}=$ 20.27 (or approximately 20 reaction zone lengths), where the relaxation length is defined as the distance taken to be within $5 \%$ of the final steady state shock pressure after the interface.

Figures 4.2 and 4.3 show $x$ - $t$ or distance-time numerical schlieren graphs of this process. The pressure profiles of the detonation throughout this relaxation process are superimposed on the graph in figure 4.2 and the corresponding temperature profiles in figure 4.3. The first profile (at $x / \delta=-0.5$ ) shows the detonation wave immediately before the interface (at $x / \delta=0)$. It should be noted that after the interaction, the schlieren indicates the formation of a reflected expansion wave and a contact surface that is advected with 
Profiles of pressure for $\Delta \rho=-25 \%$

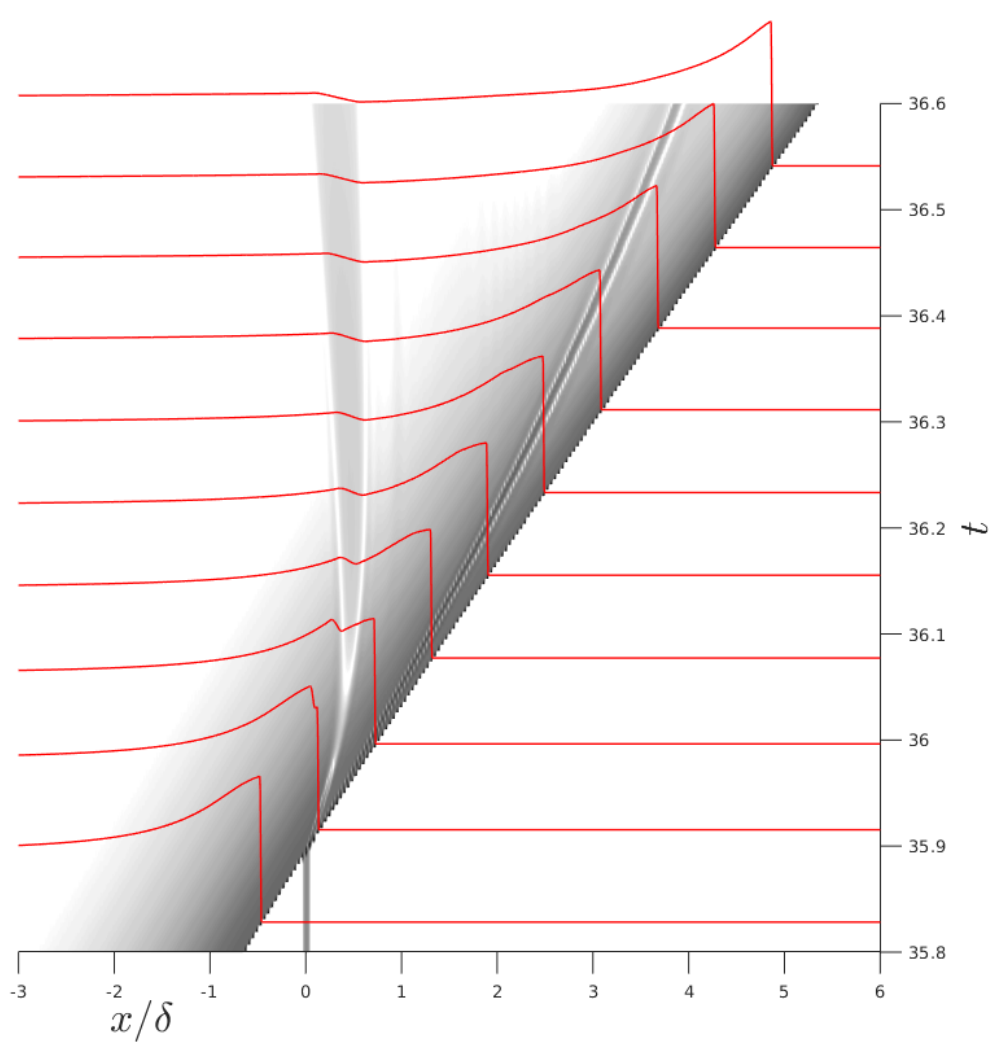

Figure 4.2: Pressure profiles and schlieren plot for $\Delta \rho=-25 \%$ and single step Arrhenius kinetics with $E_{a}=20, Q=50, \gamma=1.2$ 
Profiles of temperature for $\Delta \rho=-25 \%$

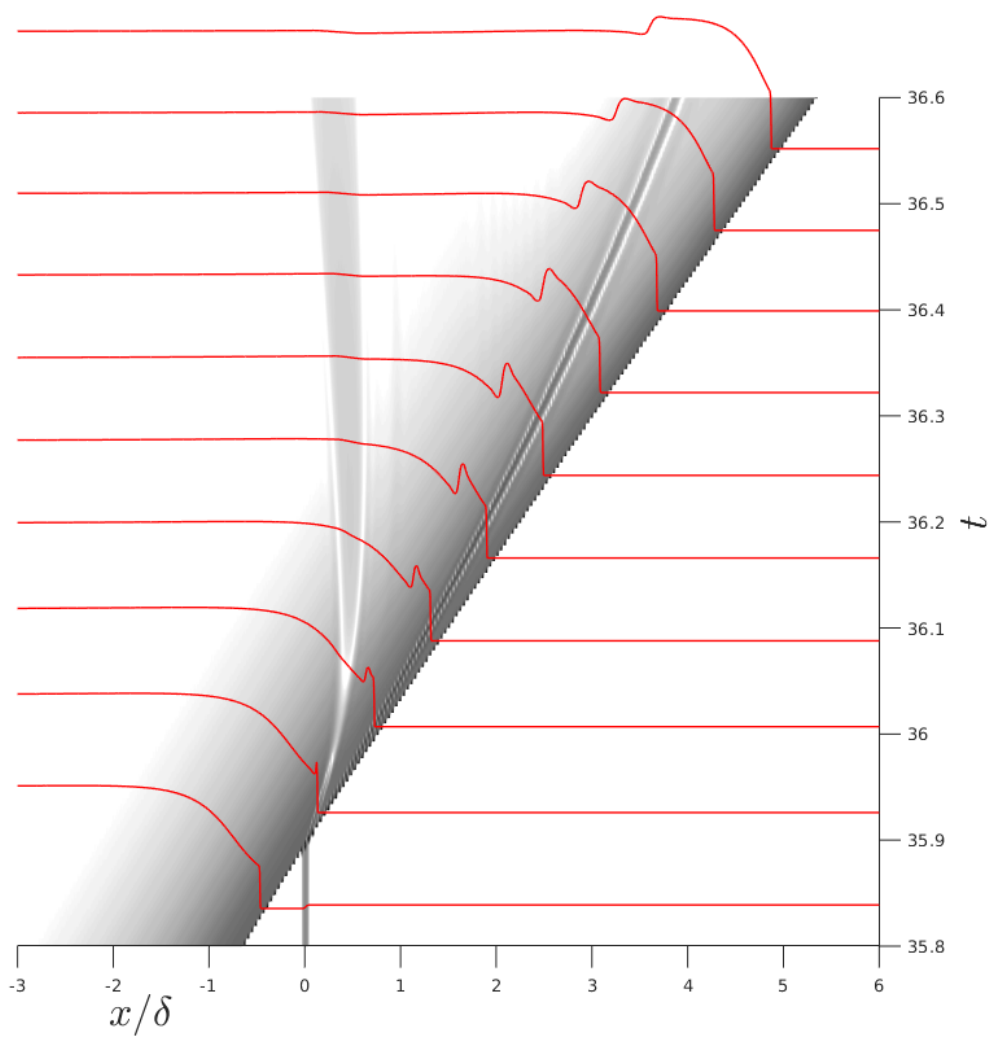

Figure 4.3: Temperature profiles and schlieren plot for $\Delta \rho=-25 \%$ and single step Arrhenius kinetics with $E_{a}=20, Q=50, \gamma=1.2$ 
the flow. This was predicted theoretically. The reflected expansion wave is evident by the pressure and temperature decrease seen in the profiles across it. Across the contact surface the profiles indicate constant pressure but a jump in temperature as expected.

The initial decrease in shock pressure is due to the interaction of the leading shock with the interface. Across the interface there is a temperature increase and thus a corresponding increase in sound speed, $c$. Consequently, there is a decrease in acoustic impedance, $z$ $=\rho c=\frac{p \gamma}{c}$ for an ideal gas. Change in acoustic impedance is an accurate predictor of the shock-interface transmission problem when $\frac{\gamma_{0}}{\gamma_{1}}<\frac{\rho_{1}}{\rho_{0}}<\frac{\gamma_{0}+1}{\gamma_{1}+1}$, otherwise the shock impedance must instead be considered [24]. In this study $\gamma$ is assumed constant and so the acoustic impedance is always an accurate indicator. A shock wave encountering this interface with decreased acoustic impedance will generate a reflected expansion wave and suffer an abrupt decrease in shock pressure $\left(\frac{p_{+}}{p_{-}}\right)[25]$. Here $\frac{p_{+}}{p_{-}}$decreases to 35.56. An inert shock at the same initial pressure encountering the same density decrease has a transmitted shock pressure of $\frac{p_{+}}{p_{-}}=35.41$. Thus, this initial decrease is approximately $0.4 \%$ different to the inert shock case. Details of this calculation are given in Appendix B.

Although the pressure at the shock front has decreased, the flow behind the shock is still at its initial pressure, therefore creating a high pressure region behind the shock. For the detonation wave, this corresponds to the second profile of figure 4.2 where the region of high pressure from behind the incident detonation remains. This high pressure behind the shock must expand via a reflected expansion wave seen in subsequent profiles around $\frac{x}{\delta}=0.5$. We can see that by $\frac{x}{\delta}>1$ the high pressure region has been almost fully expanded.

Comparing the incident $(i)$ to the transmitted $(t)$ detonation, the shock temperature ratio, $\frac{T_{+, t}}{T_{-, t}}<\frac{T_{+, i}}{T_{-, i}}$ since $M_{D, t}<M_{D, i}$. However, there has been a temperature increase across the interface, such that the temperature in front of the transmitted detonation 
Profiles of reation rate for $\Delta \rho=-25 \%$

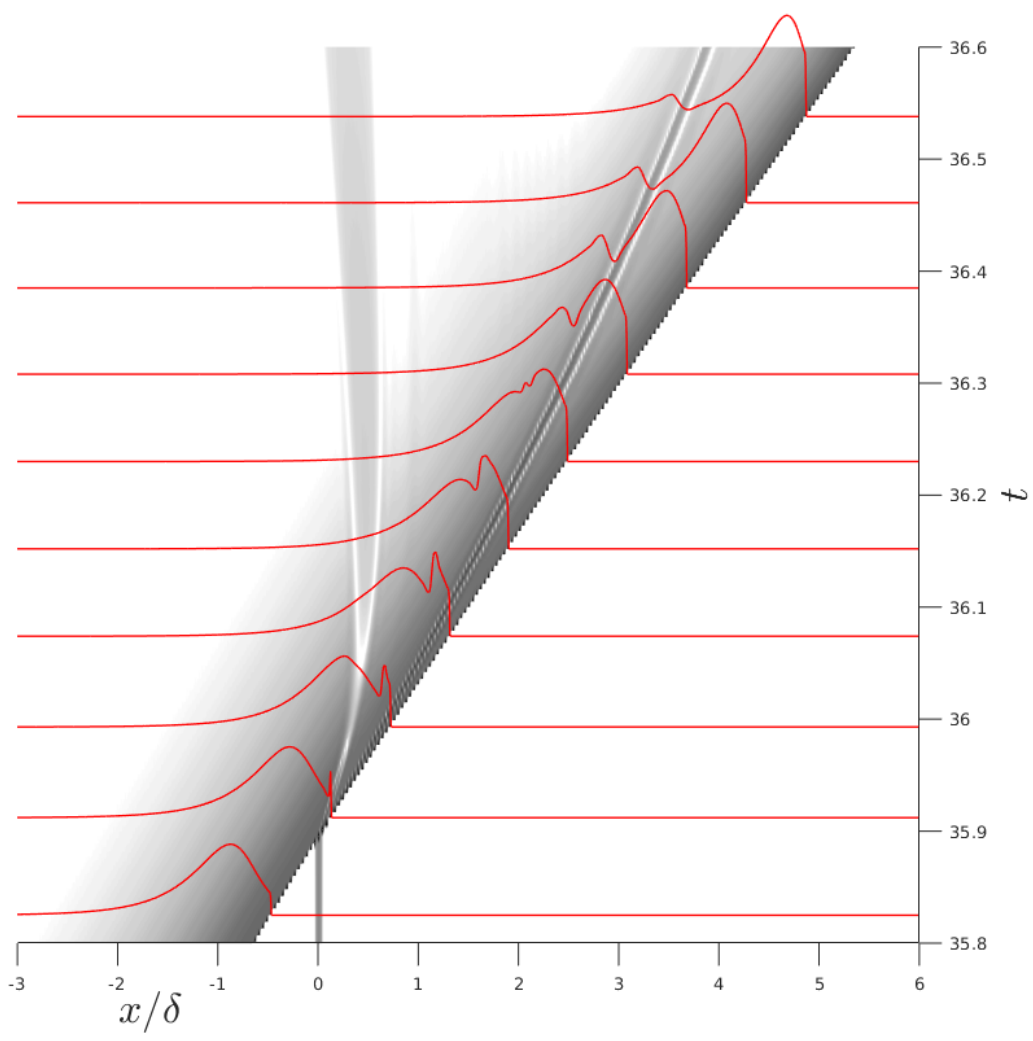

Figure 4.4: Reaction rate, $\dot{\omega}$ and Schlieren plot for $\Delta \rho=-25 \%$ and single step Arrhenius kinetics with $E_{a}=20, Q=50, \gamma=1.2$

$T_{-, t}>T_{-, i}$. This overcomes the decreased temperature ratio and the shock temperature is overall increased, $T_{+, t}>T+, i$. This can be seen on the second temperature profile in figure 4.3.

The reaction rate is an exponential function of temperature (equation 3.3), thus the increased shock temperature $T_{+}$begins to cause an increase in the reaction rate behind the shock front. Beginning around $\frac{x}{\delta} \sim 1$, this can also be seen in figure 4.4 where profiles showing the reaction rate $\dot{\omega}=\frac{d \lambda}{d t}$ are plotted on the same $x-t$ diagram.

The contact surface separates gas that has already mostly reacted on the left of the interface from the fresh reactants at higher temperature on the right. Initially it is seen 


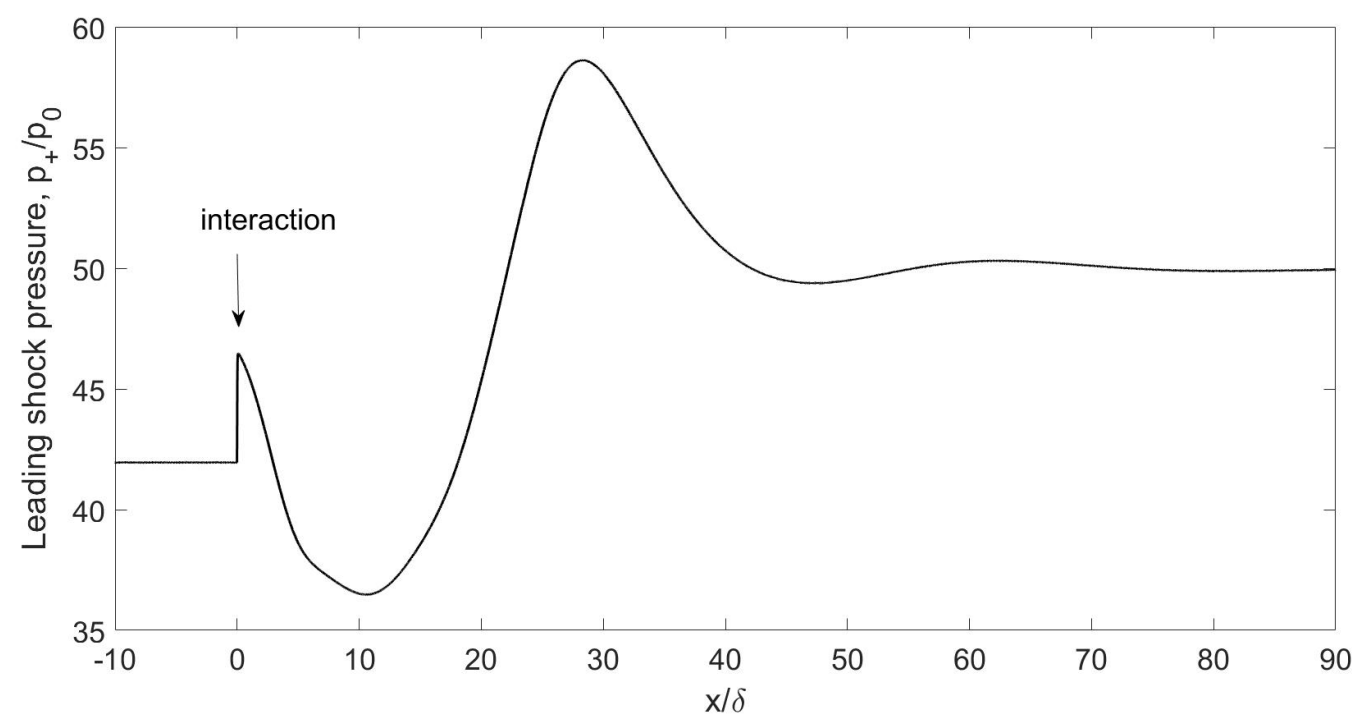

Figure 4.5: Relaxation process for $\Delta \rho=20 \%$ with single step Arrhenius kinetics with $E_{a}=20, Q=50$ and $\gamma=1.2$

that there is chemical energy release on both sides of the contact surface. As the distance between the contact surface and shock front increases in subsequent profiles, the gas originally at the left of the interface is burnt out. However, simultaneously, fresh unreacted gas at increasingly higher shocked temperature enters from the right, causing further increase in reaction rate. Thus, increased rate of chemical energy release leads to acceleration of the wave.

At $\frac{x}{\delta} \sim 4.8$, the reaction rate has stopped increasing (figure 4.4) and the detonation asymptotically decays to the final state. This is also demonstrated in the pressure profiles (figure 4.2), where during the acceleration phase the reaction zone become more curved as pressure builds due to energy release.

\subsubsection{Density increase}

Now consider density increase and temperature decrease across the interface.

Figure 4.5 shows the variation of the leading shock pressure, $p_{+}$with distance, $x$. 
Pressure is normalized by the initial pressure of the unburnt material, $p_{0}$ and distance is normalized with reaction zone length, $\delta$. The $x$-axis is shifted such that the interface is located at $\frac{x}{\delta}=0$. A density increase of $\Delta \rho=20 \%$ and the single step Arrhenius model are used with $E_{a}=20, Q=50$ and $\gamma=1.2$.

Upon interaction there is an abrupt increase of $\sim 10 \%$ in shock pressure ratio to $\frac{p_{+}}{p_{0}}=\frac{p_{+}}{p_{-}} \sim 46.5$. This is followed immediately by decay to a sub-CJ state of $\sim 85 \%$ of the CJ detonation velocity, $D_{C J}$ at the right of the interface. There is a quasi-steady period $(\sim 10$ reaction zone lengths $\delta)$ at this low velocity. Subsequently, the wave re-accelerates to a shock pressure ratio $\frac{p_{+}}{p_{-}} \sim 58.6$ and then asymptotically decays to the final state the at CJ value. This relaxation length is $\frac{x}{\delta}=36.93$ (or $\sim 37$ reaction zone lengths), where the relaxation length is defined as the distance taken to be within $5 \%$ of the final shock pressure downstream of the interface.

Figures 4.6 and 4.7 show $x$ - $t$ or distance-time numerical schlieren graphs of this process. The pressure profiles of the detonation throughout this relaxation process are superimposed onto the plot in figure 4.6 and the corresponding temperature profiles in 4.7. The first two profiles show the stable detonation wave immediately before the interface (at $x / \delta=0)$. It can be noted that after the interaction, the Schlieren indicates the formation of a reflected shock wave and a contact surface. This was predicted theoretically. The reflected shock wave is evident by the pressure and temperature increase seen in the profiles across it. Across the contact surface the profiles indicate constant pressure but a jump in temperature as expected.

The abrupt increase in leading shock pressure, $p_{+}$is due to the interaction of the leading shock with the interface. Across the interface there is a temperature decrease and thus a corresponding decrease in sound speed, $c$. Consequently, there is an increase in acoustic impedance, $z=\rho c=\frac{p \gamma}{c}$ for an ideal gas. As previously discussed, for this problem acoustic impedance is an accurate indicator of the shock-interface transmission 
Pressure for $\Delta \rho=+20 \%$

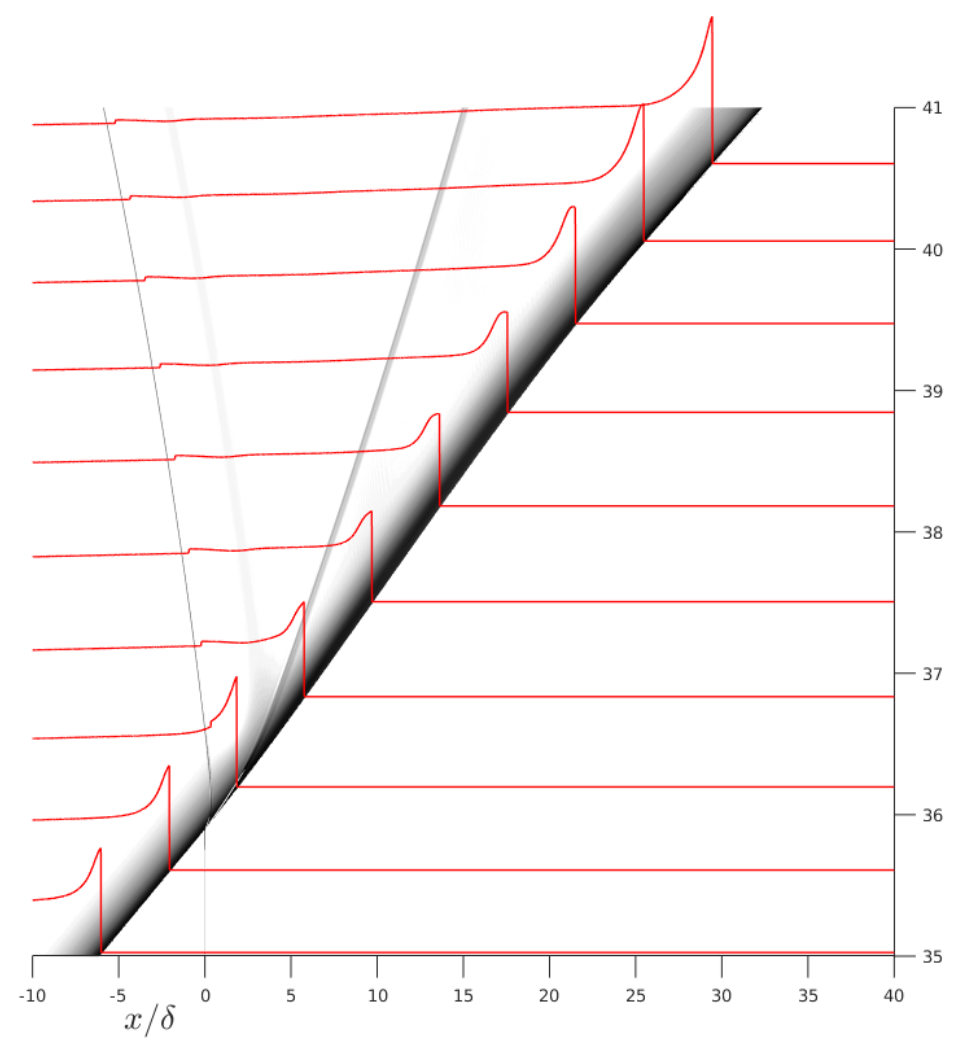

Figure 4.6: Pressure profiles and Schlieren plot for $\Delta \rho=20 \%$ and single step Arrhenius kinetics with $E_{a}=20, Q=50, \gamma=1.2$ 
Temperature for $\Delta \rho=+20 \%$

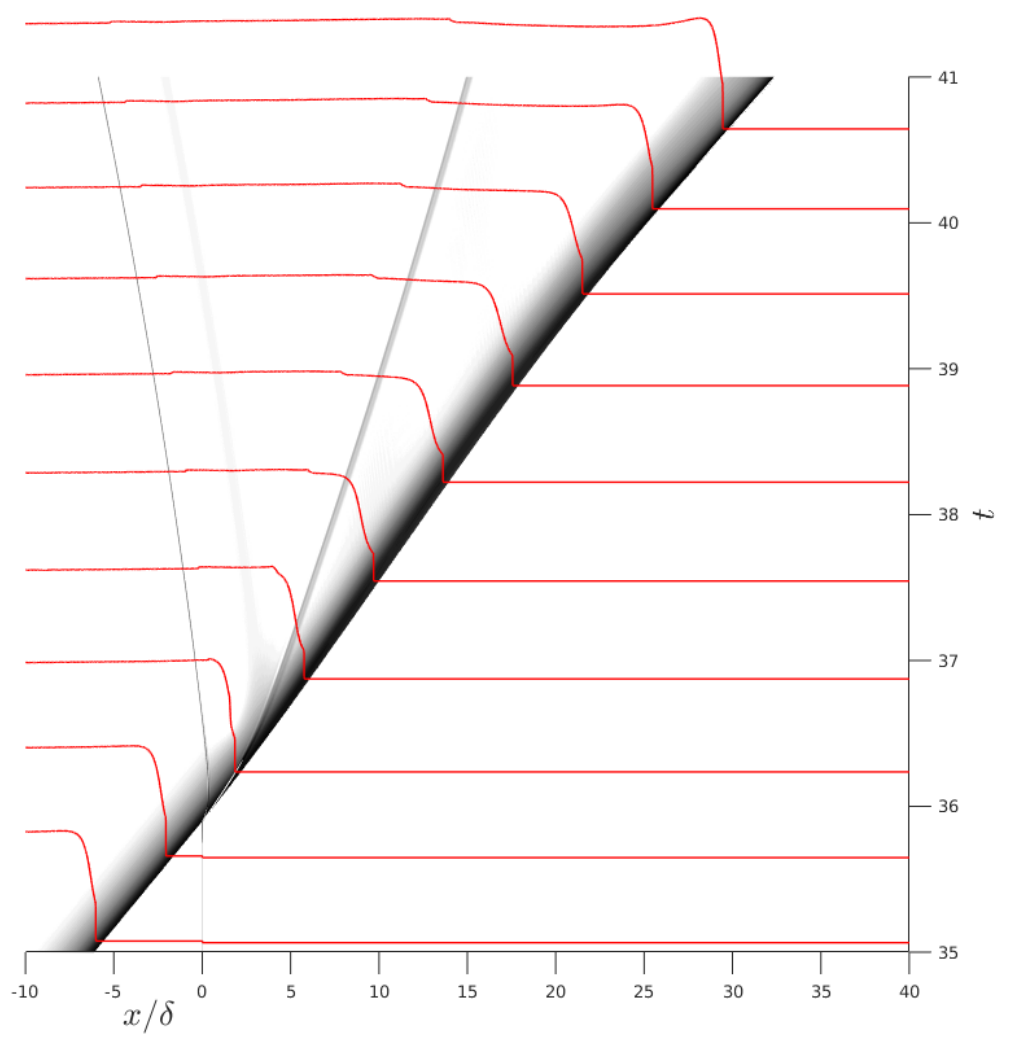

Figure 4.7: Temperature profiles and Schlieren plot for $\Delta \rho=20 \%$ and single step Arrhenius kinetics with $E_{a}=20, Q=50, \gamma=1.2$ 
problem. This increase implies the formation of a reflected shock wave and an increased transmitted shock pressure $\frac{p_{+}}{p_{-}}[25]$. Here $\frac{p_{+}}{p_{-}}$increases to 46.48 . An inert shock at the same initial pressure encountering the same density change has a transmitted shock pressure $\frac{p_{+}}{p_{-}}=46.61$. Thus, this initial increase is approximately $0.3 \%$ different to the inert shock case. Details of this calculation are given in Appendix B.

Although detonation Mach number has increased $M_{D, t}>T_{D, i}$, there is lower temperature on the right of the interface in front of the transmitted detonation $T_{-, t}<T_{-, i}$. This leads to a decrease in shock temperature, $T_{+, t}<T_{+, i}$. The reaction rate is an exponential function of temperature (equation 3.3), thus there is a decrease in reaction rate and consequently energy release behind the leading shock causing its immediate decay. However, there is still some energy release in the reaction zone, slowing its deceleration and eventually leading to its quasi-stabilization.

This is illustrated in figure 4.8 where profiles showing the reaction rate $\dot{\omega}=\frac{d \lambda}{d t}$ are plotted on the same $x$-t diagram. Before the interface at $\frac{x}{\delta}=0$, the point of maximum reaction rate is near to the shock front. After the interface, this local maximum begins to decrease and moves away from the shock front, effectively causing its deceleration. However, by $\frac{x}{\delta} \sim 10$, or 10 reaction zone lengths downstream, energy release begins increase again and re-accelerates the wave. The maximum reaction rate increases and moves forward to again become coupled with the shock front. At this point $\left(\frac{x}{\delta} \sim 30\right)$, the wave stops accelerating and asymptotically decays to the final state.

This process is analogous to direct initiation for a detonation in one dimension [11]. From the pressure profiles in figure 4.6 it can also be seen that the increasing energy release causes pressure increase in the reaction zone that re-accelerates the leading shock due to the inverse pressure gradient. 


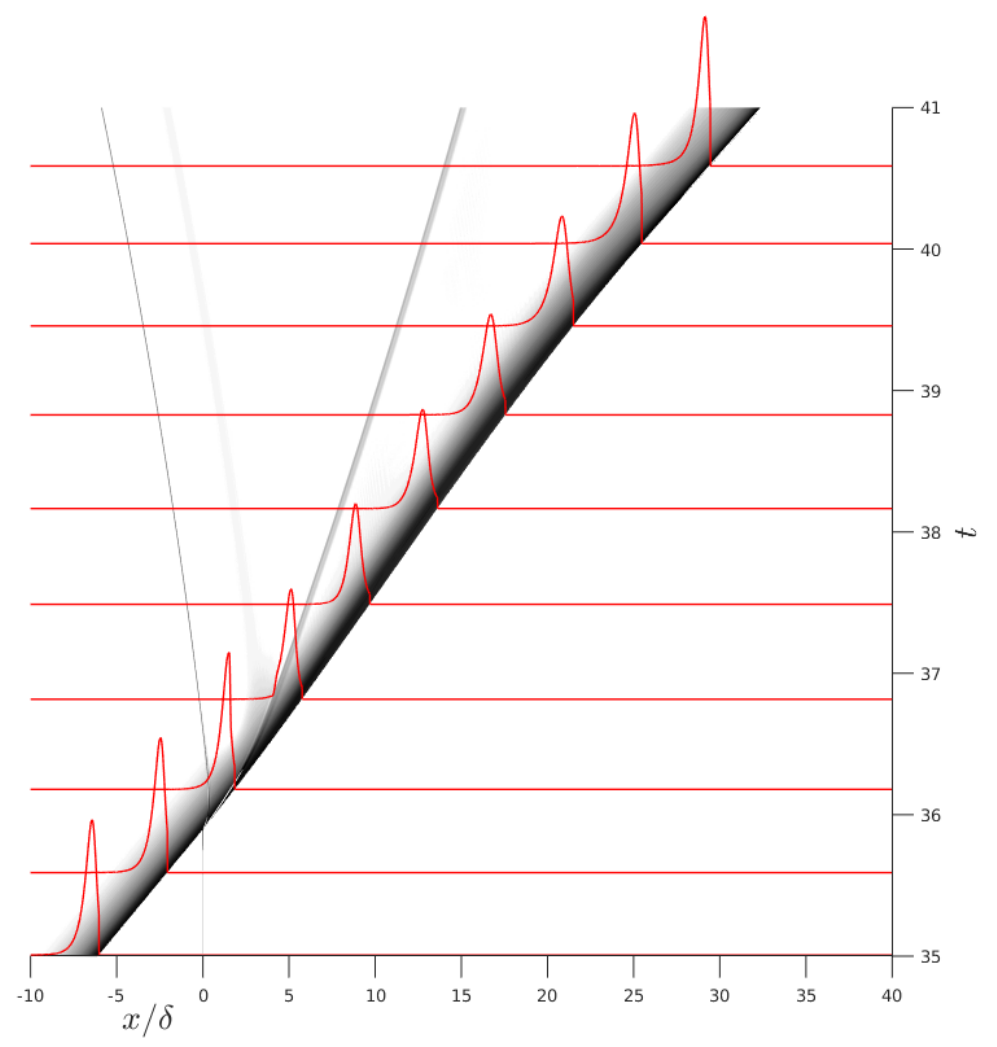

Figure 4.8: Reaction rate, $\dot{\omega}$ and Schlieren plot for $\Delta \rho=20 \%$ and single step Arrhenius kinetics with $E_{a}=20, Q=50, \gamma=1.2$ 


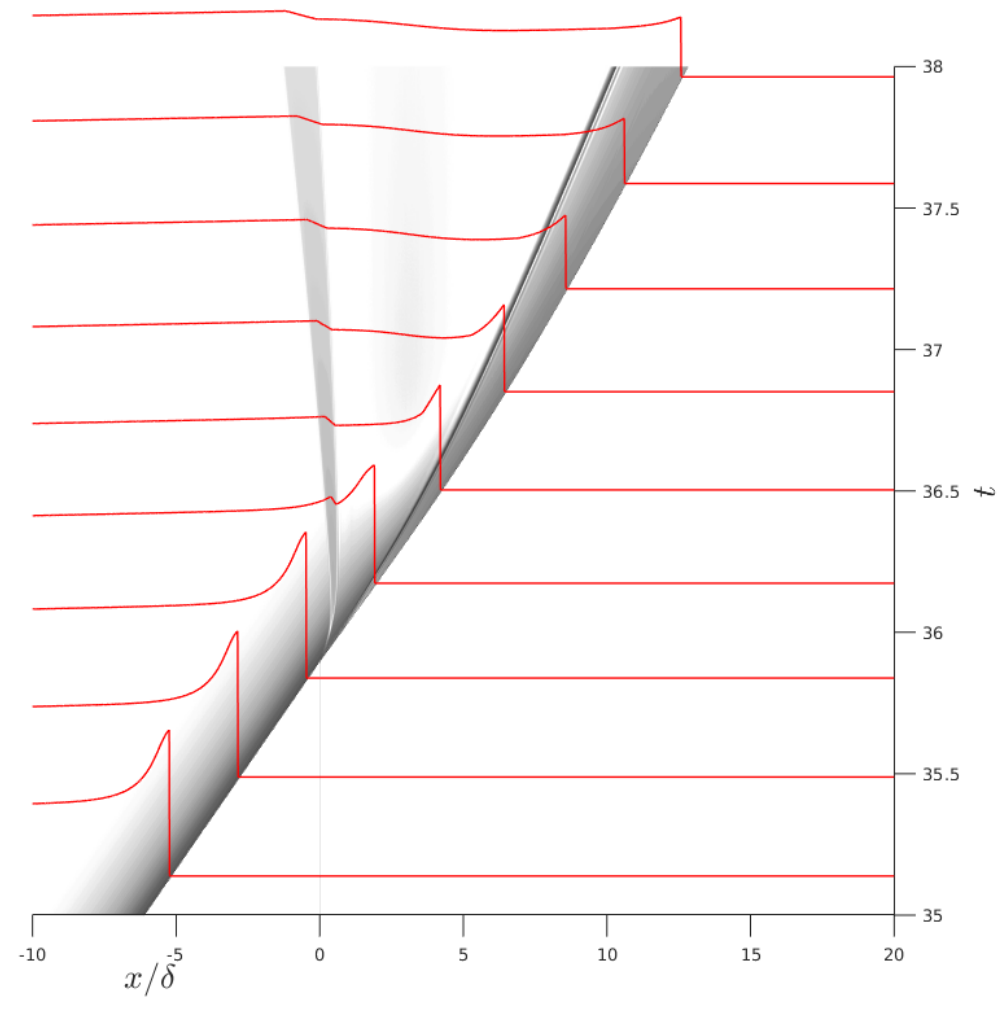

Figure 4.9: Pressure and Schlieren plot for single step Arrhenius kinetics with $E_{a}=20$, $Q=50, \gamma=1.2$ to an inert gas where $\Delta \rho=-25 \%$

\subsubsection{Inert downstream medium}

It is perhaps worthwhile to compare these to a case with the same density change, but where the downstream medium is inert (i.e. $Q=0$ or $\lambda=0$ ). In this case, the transmitted detonation does not have the supporting energy release and decays into a transmitted shock wave.

The figures 4.9 and 4.10 show the pressure profiles for these cases superimposed on an $x$ - $t$ numerical schlieren graph as before. The single step Arrhenius model is used with $E_{a}=20, Q=50$ and $\gamma=1.2$ on the left of the interface. Initially, downstream of the 
Pressure for $\Delta \rho=+20 \%$

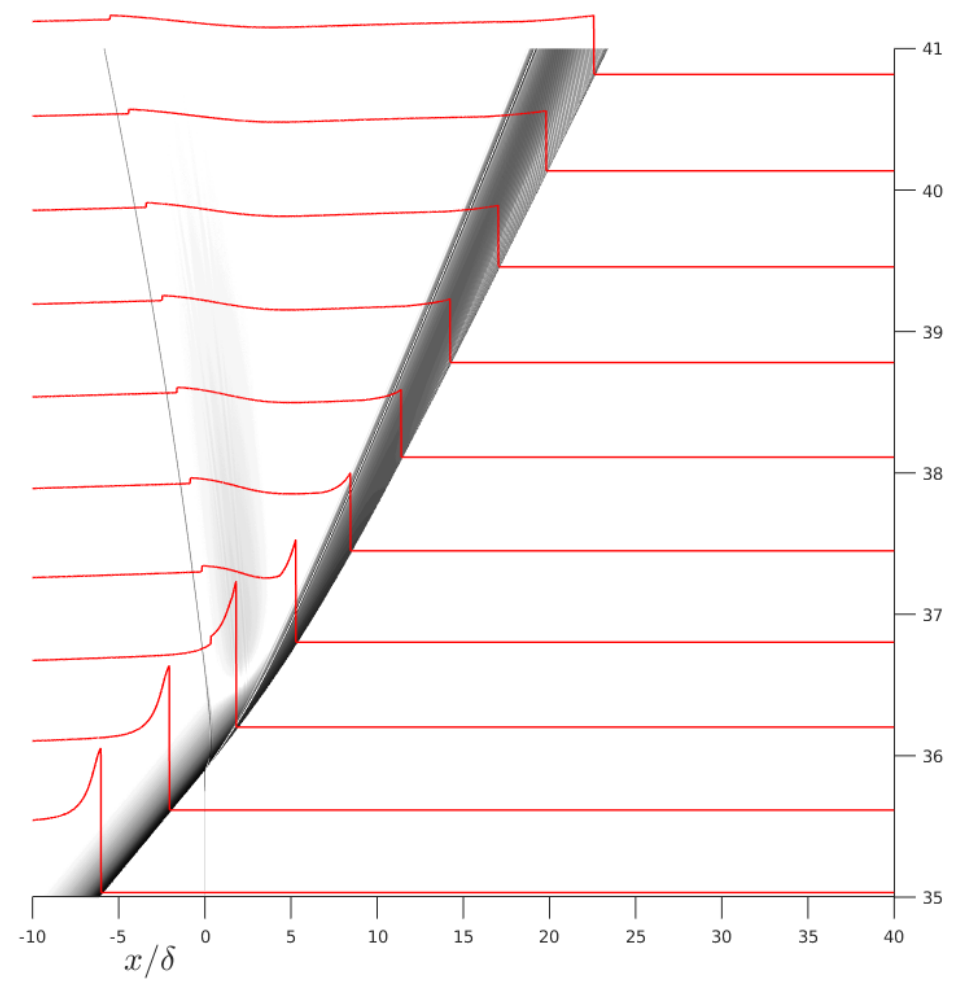

Figure 4.10: Pressure and Schlieren plot for single step Arrhenius kinetics with $E_{a}=20$, $Q=50, \gamma=1.2$ to an inert gas where $\Delta \rho=20 \%$ 
interface $\Delta \rho=-25 \%, 20 \%$ and $\lambda=0$ (inert).

Once the detonation transmits across the interface at $\frac{x}{\delta}=0$, there is still some partially burnt gas in the reaction zone, originating from the left of the interface. However, the remaining reactant is eventually burnt out and a transmitted shock wave is formed.

As seen for the density decrease schlieren of figure 4.9, a reflected expansion wave is formed similarly to that in the case a reactive downstream medium. In the same way for the density increase schlieren of figure 4.10 a reflected shock wave is seen to be formed. A contact surface advects with the flow in both cases as before.

\subsection{Effect of $\Delta \rho$}

The transient dynamics and effect of chemical energy release are further investigated by varying the density change, $\Delta \rho$.

\subsubsection{Density decrease}

The initial ZND wave is held constant with $E_{a}=20, Q=50, \gamma=1.2$ and single-step Arrhenius kinetics. A change in density $\Delta \rho$ of $-15 \%,-25 \%$ and $-35 \%$ is used. The simulation results are compared in figure 4.11 by plotting the change in leading shock pressure, where $p_{+}$for each $\Delta \rho . p_{+}$is normalized with the initial unburnt pressure $p_{0}$, and the $x$-axis for each plot is normalized with the reaction zone length, $\delta$. The incident detonation is the same in each case and thus $\delta$ is the same.

The relaxation processes for different $\Delta \rho$ are seen to be qualitatively similar as the wave goes through the same transient processes. The shock acceleration is seen to occur for approximately 5 reaction zone lengths, $\delta$ in each case. However, it is clear that as the magnitude of $\Delta \rho$ increases, the wave acceleration phase becomes steeper.

Recall that as $|\Delta \rho|$ increases, $|\Delta T|$ also increases. This implies decreased temperature 


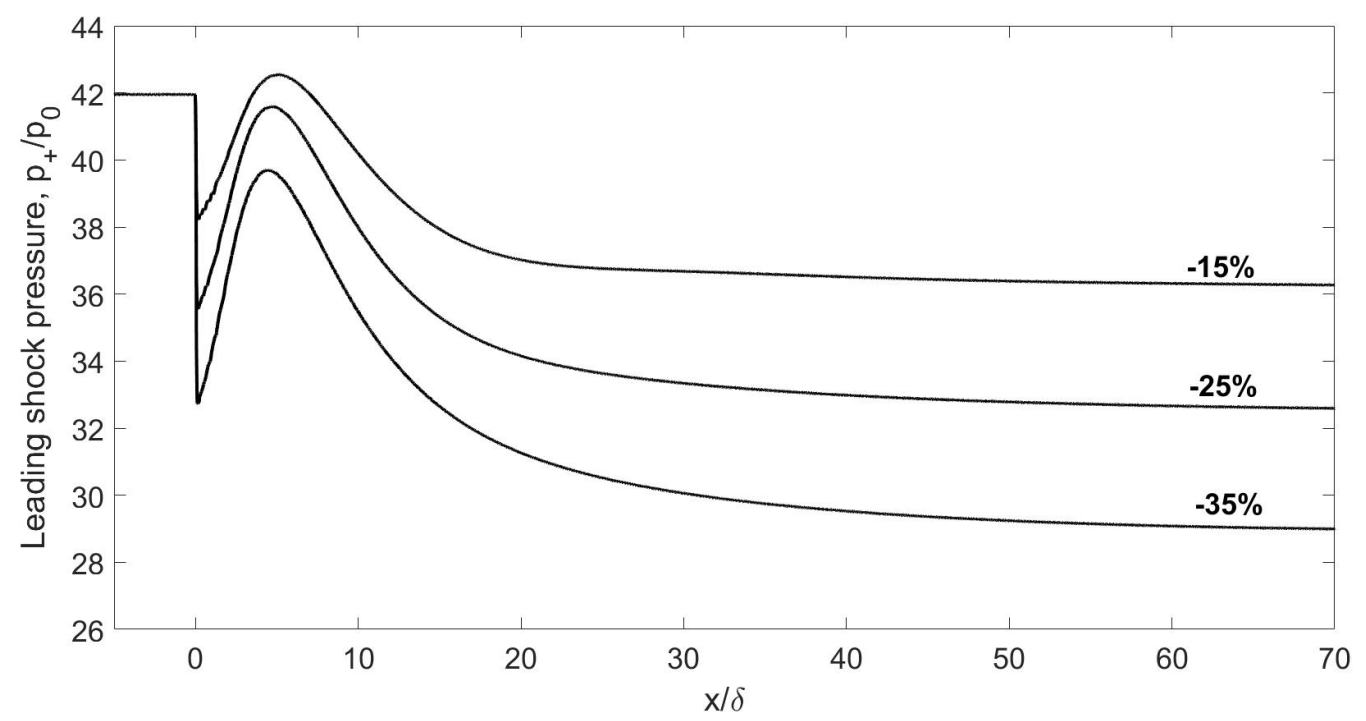

Figure 4.11: Relaxation process for single step Arrhenius kinetics with $E_{a}=20, Q=50$, $\gamma=1.2$ and $\Delta \rho=-15 \%,-25 \%,-35 \%$

after the interface in front of the transmitted detonation, $T_{-, t}$. This leads to a larger shock temperature of the transmitted detonation after the interface $T_{+, t}$. Reaction rate is an exponential function of temperature (equation 3.3), and this larger shock temperature change causes a more rapid change in reaction rate. Thus, increased $\Delta \rho$ leads to a more rapid increase in reaction rate and thus a more rapid acceleration.

\subsubsection{Density increase}

As for the density decrease case, the initial ZND wave is held constant with $E_{a}=20$, $Q=50, \gamma=1.2$ and single step Arrhenius kinetics. This time, a change in density $\Delta \rho$ of $+10 \%,+20 \%$ and $+30 \%$ is used.

Figure 4.12 shows the change in leading shock pressure $p_{+}$for these cases. Increased $\Delta \rho$ implies increased $\Delta T$ and increased temperature in front of the transmitted detonation $T_{-, t}$. This leads to a larger decrease in shock temperature of the transmitted detonation, $T_{+, t}$. Reaction rate is an exponential function of temperature (equation 3.3), so this larger 


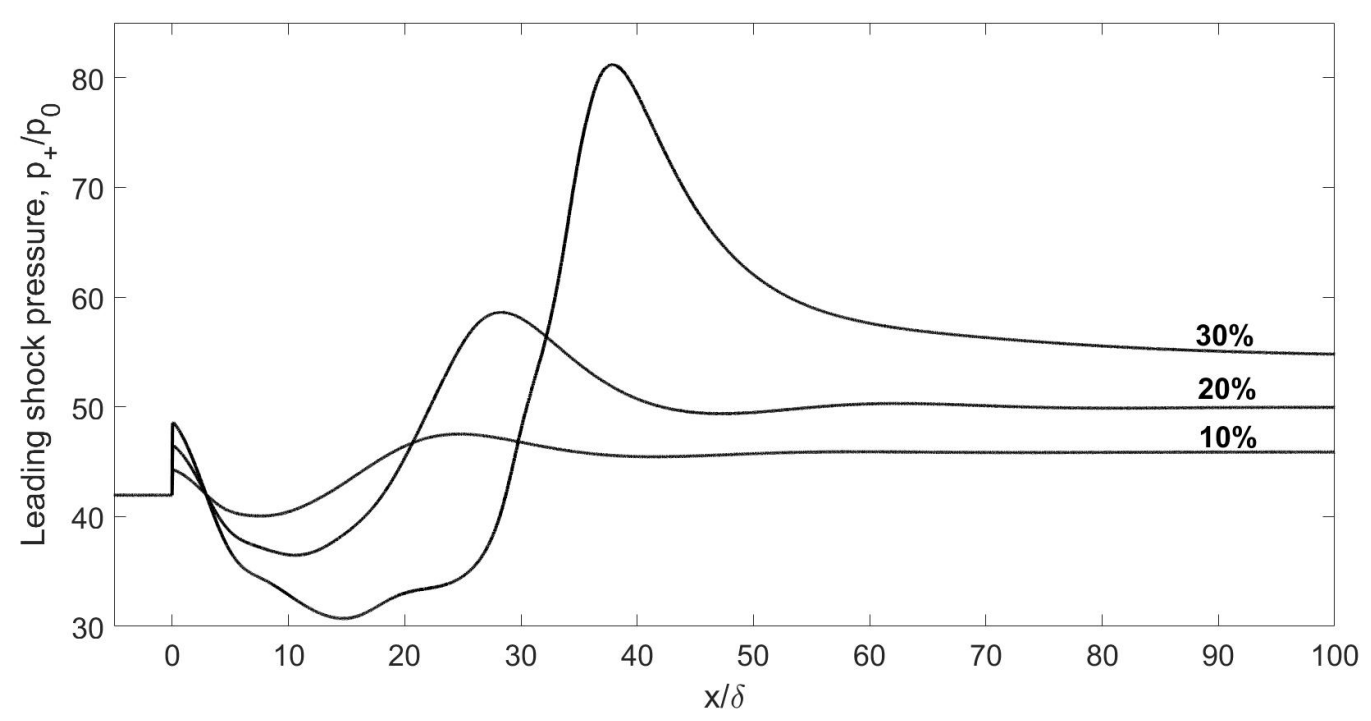

Figure 4.12: Relaxation process for single step Arrhenius kinetics with $E_{a}=20, Q=50$, $\gamma=1.2$ and $\Delta \rho=+10 \%,+20 \%,+30 \%$

temperature change causes a more rapid change in reaction rate. Thus, the deceleration and acceleration phases are seen to be steeper for a larger $\Delta \rho$.

Reduced activation energy is defined as $\tilde{E}_{a}=\frac{E_{a}}{R T}$, where $T$ is the temperature in front of the wave, $T_{-}$in this case. As $\Delta \rho$ increases, $T_{-}$decreases and thus $\tilde{E}_{a}$ increases across the interface. This increase in $\tilde{E}_{a}$ implies greater inherent instability of the detonation. This is evident in the low velocity region of figure 4.11 where for increasing $\Delta \rho$ some oscillations occur.

\subsection{Effect of kinetic model}

The effect of the kinetic model on the transient process was also studied, specifically the addition of an induction zone. To this end, the simulations were repeated using the two step induction-reaction kinetic model. 


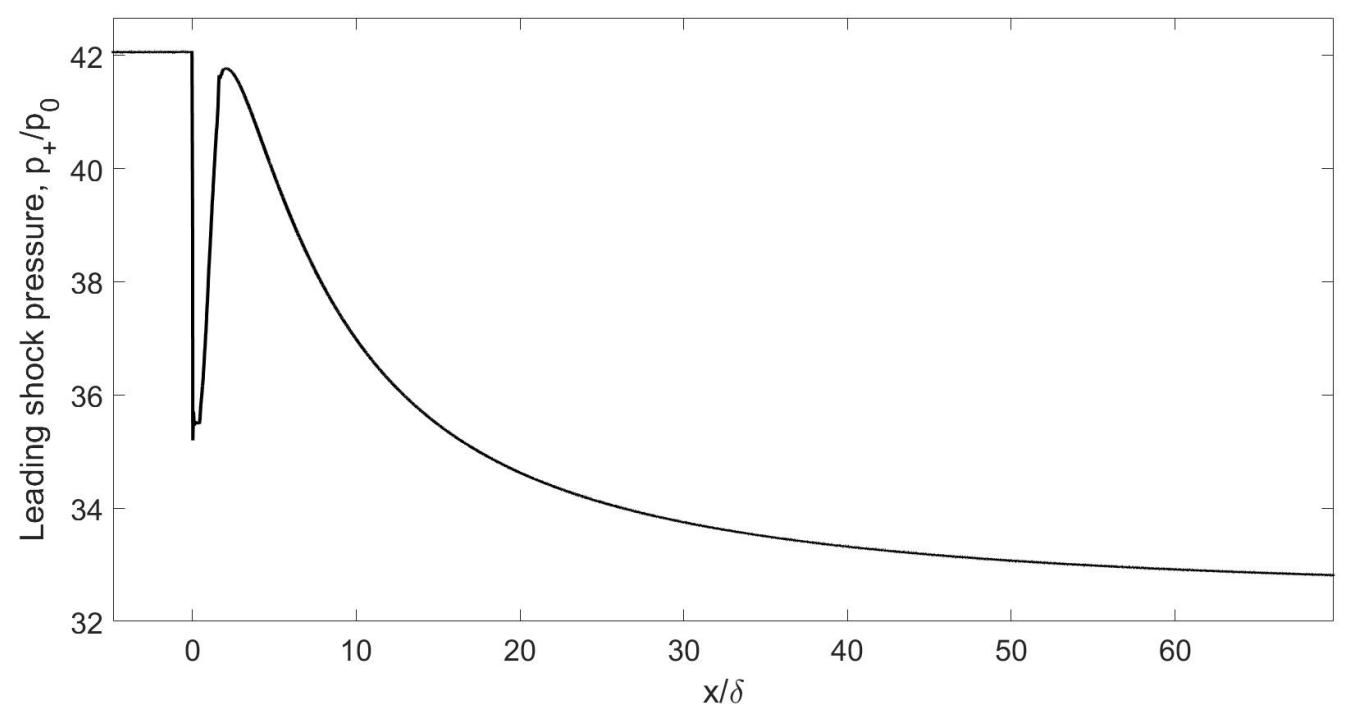

Figure 4.13: Relaxation process for the two step kinetic model with $\epsilon_{i}=8, \epsilon_{r}=1$, $k_{i}=0.779, k_{r}=0.887, Q=50, \gamma=1.2$ and $\Delta \rho=-25 \%$

\subsubsection{Density decrease}

Qualitatively similar results are obtained using the two step induction-reaction model with the same density decrease $\Delta \rho=-25 \%$ as in section 4.1.1. The change in leading shock pressure, $p_{+}$is plotted in figure 4.13 , where $\epsilon_{i}=8, \epsilon_{r}=1, k_{r}=0.887, Q=50$ and $\gamma=1.2$. $k_{i}$ is the particle velocity behind the shock front in the shock-fixed frame, here found to be 0.779 . As before, $p_{+}$is normalized with respect to the initial unburnt pressure $p_{0}$ and $x$ is normalized by the reaction zone length, $\delta . \delta$ is defined as the location of $\lambda=0.1$. Thus, the detonation wave goes through the same transient physical processes in response to the change.

However, for the two step induction-reaction model there is a brief delay $\left(\frac{x}{\delta} \sim 0.5\right)$ before the shock acceleration phase due to the presence of the induction zone. This is not present for the single step Arrhenius model, see figure 4.1. The increased shock temperature for the transmitted detonation $T_{+, t}$ (as a result of increased initial temperature $T_{-, t}$ ) cannot immediately impact the reaction zone as in the single step model. It first causes 


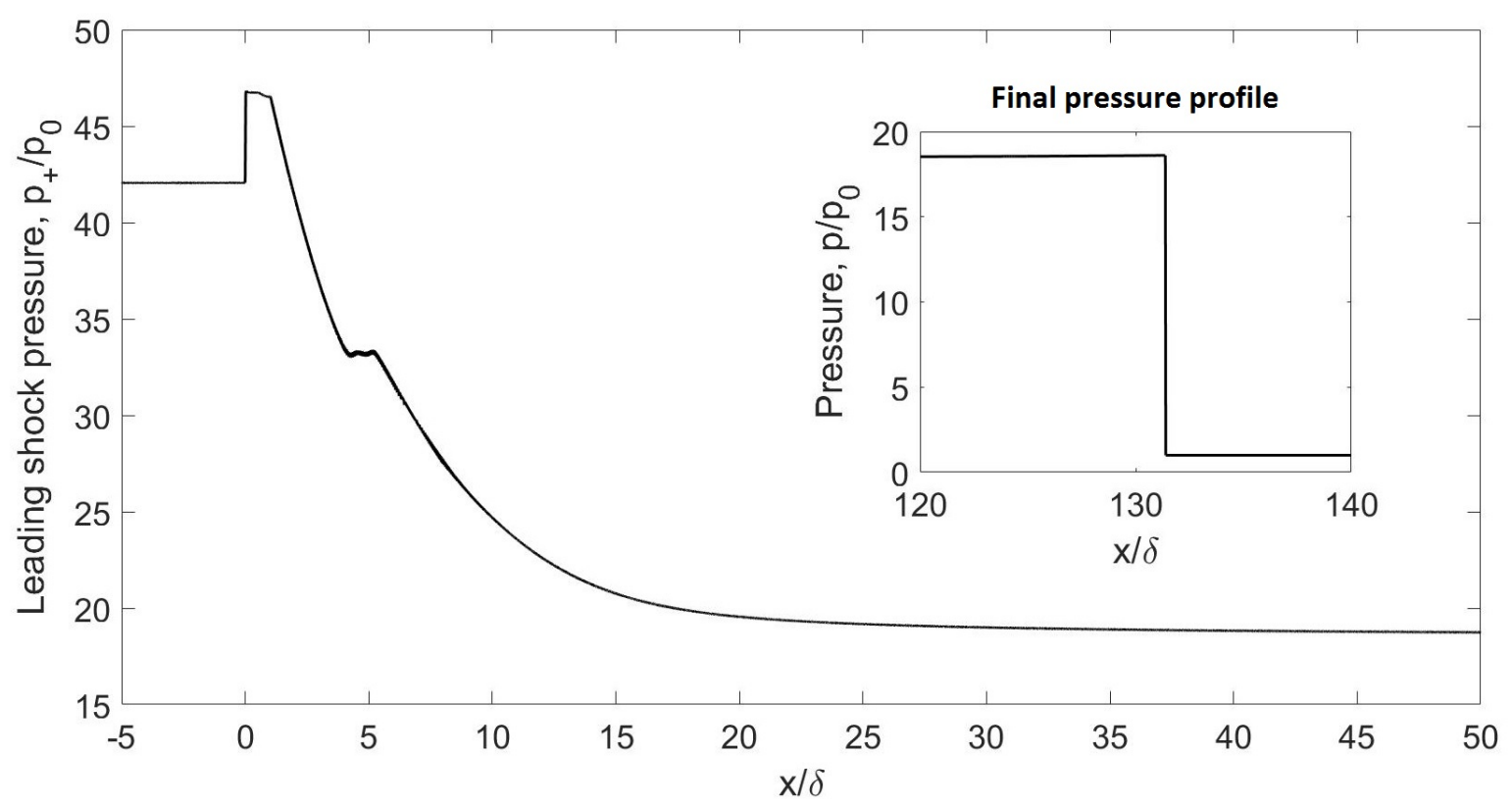

Figure 4.14: Relaxation process for the two step kinetic model with $\epsilon_{i}=8, \epsilon_{r}=1$, $k_{i}=0.779, k_{r}=0.887, Q=50, \gamma=1.2$ and $\Delta \rho=+20 \%$

an increase in the rate of change of the induction zone progress variable $\frac{d \xi}{d t}$ (equation 3.4). This decreases the induction zone length before impacting the energy release rate in the reaction zone and accelerating the wave.

\subsubsection{Density increase}

Figure 4.14 shows the change in leading shock pressure, $p_{+}$for the same $\Delta \rho=+20 \%$ as in section 4.1.2 using the two step induction-reaction kinetic model. Again $\epsilon_{i}=8$, $\epsilon_{r}=1, k_{i}=0.779, k_{r}=0.887, Q=50$ and $\gamma=1.2$.

The decreased shock temperature for the transmitted detonation, $T_{+, t}$ after the interface causes a decrease in the rate of change of the induction zone progress variable $\frac{d \xi}{d t}$ (equation 3.4). This lengthens the induction zone and causes the reaction zone to decouple from the leading shock. Unsupported by energy release, the detonation wave fails to re-initiate and the leading shock decays into a nonreactive shock wave. The inset in figure 
4.14 shows this pressure profile far downstream of the interface where the detonation has decayed to a shock with pressure $\frac{p_{+}}{p_{0}} \sim 18.6$.

This failure was also seen to occur for a smaller density increase of $\Delta \rho=+10 \%$. A full parametric study could be conducted in future research to determine critical conditions. 


\section{Chapter 5}

\section{Theoretical and Numerical \\ Comparison}

To compare the theoretical and numerical solution we look at the asymptotically approached final state of the numerical simulation far downstream from the interaction. The resulting transmitted detonation is found to agree with the theoretical predictions qualitatively and quantitatively.

In this chapter, for the density decrease case the numerical solution for both the single step Arrhenius and two step induction-reaction kinetic models are considered. However, for the density increase case only the numerical solution for the single step Arrhenius model is considered as the two step induction-reaction model was seen to be prone to failure (section 4.3.2).

\subsection{Qualitative comparison}

We first look at the structure of the final transmitted detonation. 

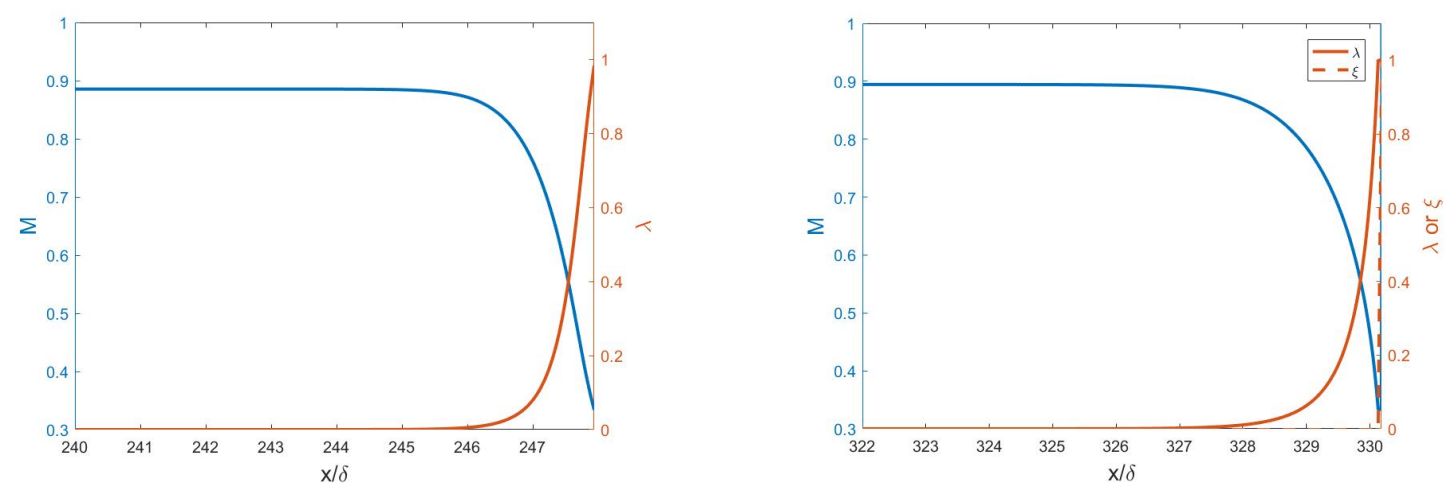

(a) Single step model with $E_{a}=20, Q=50$,(b) Two step model with $\epsilon_{i}=8, \epsilon_{r}=1, k_{i}=$ $\Delta \rho=-25 \%$ and $\gamma=1.2$

$0.779, k_{r}=0.887, Q=50, \gamma=1.2$

Figure 5.1: Transmitted detonation for density decrease $\Delta \rho=-25 \%$

\subsubsection{Density decrease}

If there is a density decrease, the transmitted detonation is found to be a strong detonation as predicted. Figure 5.1 shows the transmitted detonation obtained for $\Delta \rho=$ $-25 \%$ for the cases studied in detail in chapter 4 .

Figure 5.1a is for the single step Arrhenius chemical kinetics with activation energy $E_{a}=20$, heat release $Q=50$ and adiabatic index $\gamma=1.2$. Figure $5.1 \mathrm{~b}$ is for the two step induction-reaction kinetics with $\epsilon_{i}=8, \epsilon_{r}=1, k_{i}=0.779, k_{r}=0.887, Q=50, \gamma=1.2$

The Mach number, $M$ of the flow field in the frame of leading shock front is plotted on the left axis and the reaction progress variable or variables, $\lambda$ or $\xi$ on the right axis. The shock front is located at the right end of the $x$-axis which is normalized with respect to the reaction zone length $\delta$. This $\frac{x}{\delta}$ is measured from the initial position of the interface.

Mach number, $M$ increases through the reaction zone as $\lambda$ decreases up to a maximum of approximately 0.89 at $\lambda=0$ for both models. Thus, the models are seen to agree for the final transmitted detonation. Beyond this there is a uniform flow up to the contact surface. This subsonic product flow indicates a transmitted strong detonation as expected. 


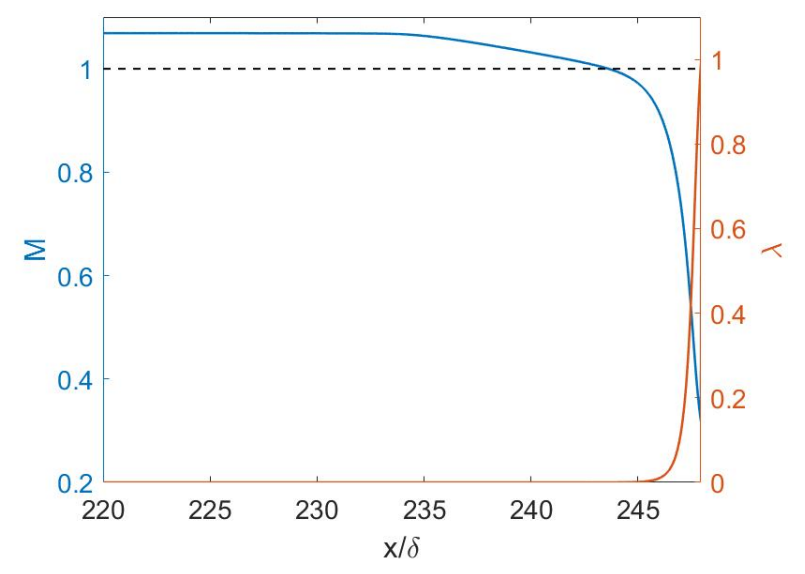

Figure 5.2: Transmitted detonation for a density increase with $E_{a}=20, Q=50, \Delta \rho=$ $+20 \%$ and $\gamma=1.2$

\subsubsection{Density increase}

If there is a density increase, the transmitted detonation is found to be CJ with an expansion wave behind it as in the theoretical solution. Figure 5.2 shows the transmitted detonation obtained for $\Delta \rho=+20 \%$ for the case studied in detail in chapter 4 . In this case the single step Arrhenius chemical kinetics are used with activation energy $E_{a}=20$, heat release $Q=50$ and adiabatic index $\gamma=1.2$.

Again the Mach number, $M$ of the flow field in the frame of leading shock front is plotted on the left axis and the reaction progress variable, $\lambda$ on the right axis. The shock front is located at the right end of the $x$-axis which is normalized with respect to the reaction zone length $\delta$. This $\frac{x}{\delta}$ is measured from the initial position of the interface. $M$ increases to a sonic $M=1$ as $\lambda$ approaches 0 at the end of the reaction zone indicated by the dashed line. Beyond this, there is further increase in $M$ behind the detonation due to the trailing expansion wave. 


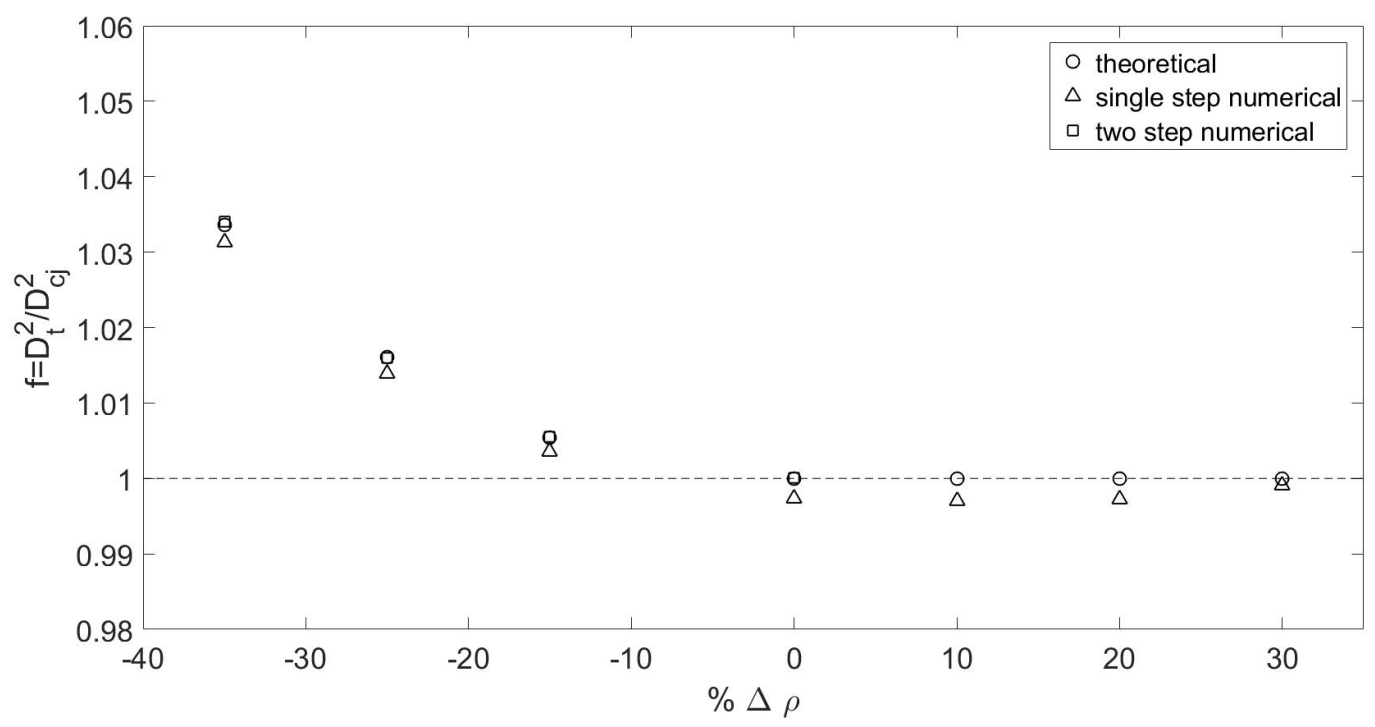

Figure 5.3: Degree of overdrive, f of the transmitted detonation for $\Delta \rho=-35 \%$ to $30 \%$. Comparison of the two kinetic models and the theoretical analysis

\subsection{Quantitative comparison}

The theoretical and downstream numerical transmitted detonation can also be compared quantitatively. In figure 5.3 the degree of overdrive, $f=\frac{D_{t}^{2}}{D_{C J}{ }^{2}}$ of the transmitted detonation is plotted against percentage density change, $\% \Delta \rho . \Delta \rho$ is varied from $-35 \%$ to $+30 \%$. Again, for the density decrease case the two kinetic models are compared with each other and the theoretical result. However, only the single step model is compared to the theoretical result for the density increase case.

For an increase in density the degree of overdrive, $f=1$, indicating a transmitted CJ detonation. Conversely, for a decrease in density the degree of overdrive, $f>1$, indicating a transmitted strong detonation. Degree of overdrive, $f$ increases with increased magnitude of density decrease.

Numerical results for both models are seen to agree with the theoretical predictions within $0.3 \%$. For the single step Arrhenius kinetic model, the largest deviation of $f$ from the theoretical was found to be $\sim 0.3 \%$ for $\Delta \rho=10 \%$. For the two step induction- 
reaction kinetic model, the largest deviation from the theoretical was found to be $\sim 0.03 \%$ for $\Delta \rho=-35 \%$.

Slight deviations such as these from exact theoretical values are common in numerical simulations, regardless of the presence of disturbances. See for example deviation in values at the $\Delta \rho=0$ point (no $\rho$ change). One reason could be the exponential nature of the Arrhenius form of the reaction rate law. The reaction can never fully go to completion and as such $100 \%$ of $Q$ is never actually released. Another reason could be the method of capturing the peak shock pressure used in the code. 


\section{Chapter 6}

\section{Conclusion}

The transmission of a detonation wave across an interface with a change in density and temperature was studied analytically and numerically. For a density decrease there was a transmitted strong detonation and for a density increase there was a transmitted CJ detonation with an expansion wave. Thus, it was shown that the transmitted detonation is not always CJ, but there can be a transmitted strong detonation depending on the back boundary condition.

The downstream state of the numerical solution was found to agree with the theoretical solution for the single step Arrhenius reaction model. This suggests that the transient relaxation process has no effect on the final transmitted wave pattern.

Furthermore, the two step induction-reaction model was seen to agree with both the single step Arrhenius model and the theoretical solution for a decrease in density across the interface. However, for an increase in density the detonation was seen to be prone to failure for the two step induction-reaction model.

This study could be extended to determine critical conditions for this aforementioned case. It could also be of interest to compare the effect of a gradual change in density and temperature to the abrupt interface studied here. 


\section{Appendix A}

The possible combinations of transmitted and reflected waves are summarized in the table below.

Table A.1: Possible wave configurations

\begin{tabular}{lll}
\hline case & reflected wave & transmitted detonation \\
\hline 1 & shock wave & strong or CJ with uniform flow \\
2 & shock wave & CJ with expansion wave \\
3 & expansion wave & strong or CJ with uniform flow \\
4 & expansion wave & CJ with expansion wave \\
\hline
\end{tabular}

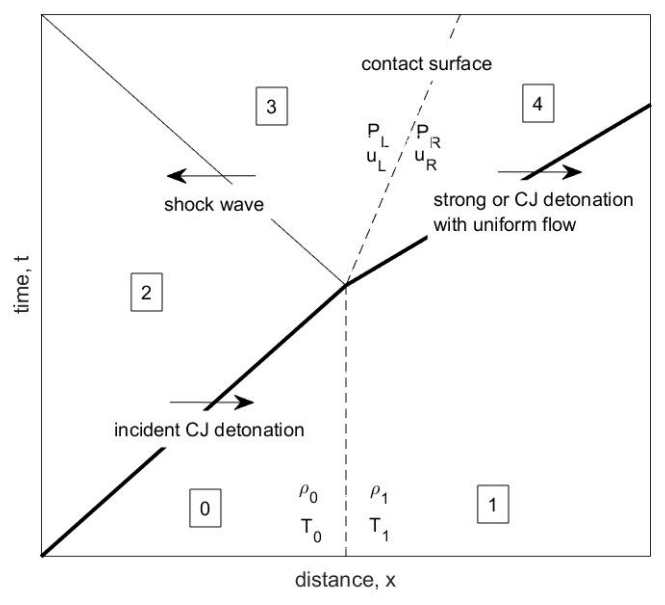

Figure A.1: Case 1: A transmitted strong or CJ detonation and a reflected shock wave 


\section{Case 1:}

Case 1 in table A.1 is a transmitted strong or CJ detonation and a reflected shock wave. It is depicted in figure A.1. As derived in section 2.2.1, the equation to be solved iteratively for case 1 is:

$$
\begin{aligned}
& \frac{c_{1}}{\sqrt{\eta_{D, t}}}\left[1-\left(\frac{\gamma+\eta_{D, t}-s}{\gamma+1}\right)\right]-u_{2} \\
& =\left(p_{2}-\frac{\eta_{D, t}+\gamma(1+s)}{\eta_{D, t}(\gamma+1)}\right)\left(\frac{\frac{2}{(\gamma+1) \rho_{2}}}{\frac{\eta_{D, t}+\gamma(1+s)}{\eta_{D, t}(\gamma+1)}+\frac{\gamma-1}{\gamma+1} p_{2}}\right)^{\frac{1}{2}}
\end{aligned}
$$

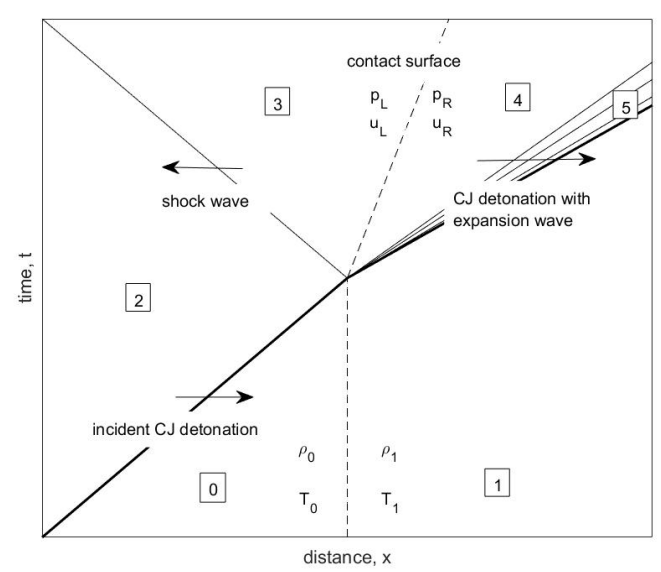

Figure A.2: Case 2: A transmitted CJ detonation with expansion wave and a reflected shock wave

\section{Case 2:}

Case 2 is a transmitted CJ detonation with an expansion wave and a reflected shock wave. It is depicted in figure A.2. The properties at 0,1 and 2 are known.

Since the transmitted detonation is CJ, this corresponds to a unique solution and the flow properties at 5 are known from equation 2.1. These can then be related to those behind the expansion wave using equation 2.2 : 


$$
u_{4}=u_{5}-\frac{2 c_{5}}{\gamma-1}\left[1-\left(\frac{p_{4}}{p_{5}}\right)^{\frac{\gamma-1}{2 \gamma}}\right]
$$

This pressure and particle velocity are equal to those on the right side of the contact surface, i.e. $p_{R}=p_{4}$ and $u_{R}=u_{4}$.

The normal shock equation, 2.3 is used to represent $p_{3}$ and $u_{3}$ behind the reflected shock:

$$
\begin{aligned}
& \frac{p_{3}}{p_{2}}=\frac{2 \gamma M_{s, r}-(\gamma-1)}{\gamma+1} \\
& u_{3}=u_{2}-\left(p_{3}-p_{2}\right)\left[\frac{\frac{2}{(\gamma+1) \rho_{2}}}{p_{3}+\frac{\gamma-1}{\gamma+1} p_{2}}\right]^{\frac{1}{2}}
\end{aligned}
$$

On the left of the contact surface $p_{L}=p_{3}$ and $u_{L}=u_{3}$.

We must have uniform pressure and particle velocity across the contact surface. Imposing $p_{L}=p_{R}$ and $u_{L}=u_{R}$ gives two equations with two unknowns ( $p_{4}$ and $\left.u_{4}\right)$ :

$$
\begin{aligned}
& u_{4}=u_{5}-\frac{2 c_{5}}{\gamma-1}\left[1-\left(\frac{p_{4}}{p_{5}}\right)^{\frac{\gamma-1}{2 \gamma}}\right] \\
& u_{4}=u_{2}-\left(p_{4}-p_{2}\right)\left[\frac{\frac{2}{(\gamma+1) \rho_{2}}}{p_{4}+\frac{\gamma-1}{\gamma+1} p_{2}}\right]^{\frac{1}{2}}
\end{aligned}
$$

These can be simultaneously solved by iteration.

\section{Case 3:}

Case 3 is a transmitted strong or CJ detonation and a reflected expansion wave. It is depicted in figure A.3. The properties at 0,1 and 2 are known.

The Rankine-Hugoniot equations, equation 2.1, are used to relate the states in front of and behind the transmitted detonation (1 and 4): 


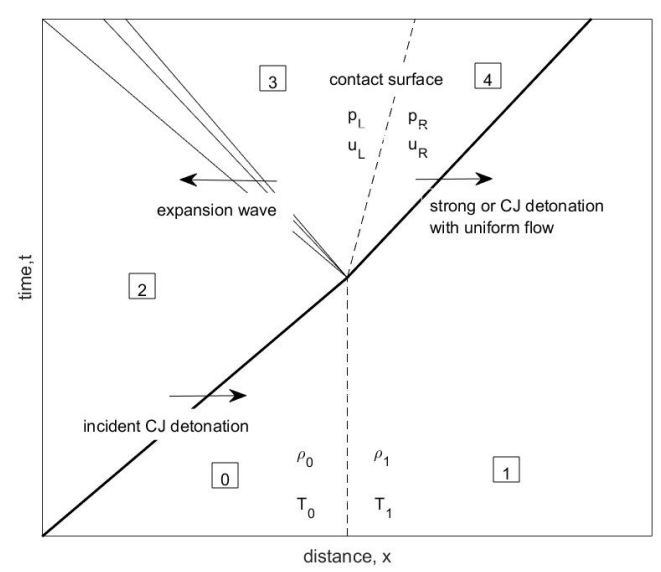

Figure A.3: Case 3: A transmitted strong or CJ detonation and a reflected expansion wave

$$
\begin{aligned}
& \frac{p_{4}}{p_{1}}=\frac{\eta_{D, t}+\gamma(1+s)}{\eta_{D, t}(1+\gamma)} \\
& u_{4}=D_{t}\left[1-\frac{\gamma+\eta_{D, t}-s}{\gamma+1}\right] \text { where } \\
& D_{t}=\frac{c_{1}}{\sqrt{\eta_{D, t}}} \\
& s=\sqrt{\left(1-\eta_{D, t}\right)^{2}-K \eta_{D, t}} \\
& K=2\left(\gamma^{2}-1\right) \frac{Q}{\gamma p_{1} v_{1}}
\end{aligned}
$$

The characteristic equation 2.4, express the change across the reflected wave from 2 to 3 :

$$
u_{3}=u_{2}+\frac{2 c_{2}}{\gamma-1}\left[1-\left(\frac{p_{3}}{p_{2}}\right)^{\frac{\gamma-1}{2 \gamma}}\right]
$$

Uniform flow behind both waves and uniform $p$ and $u$ across the contact surface imply $p_{3}=p_{4}$ and $u_{3}=u_{4}$. Thus we find an equation with one unknown $\eta_{D, t}$ : 


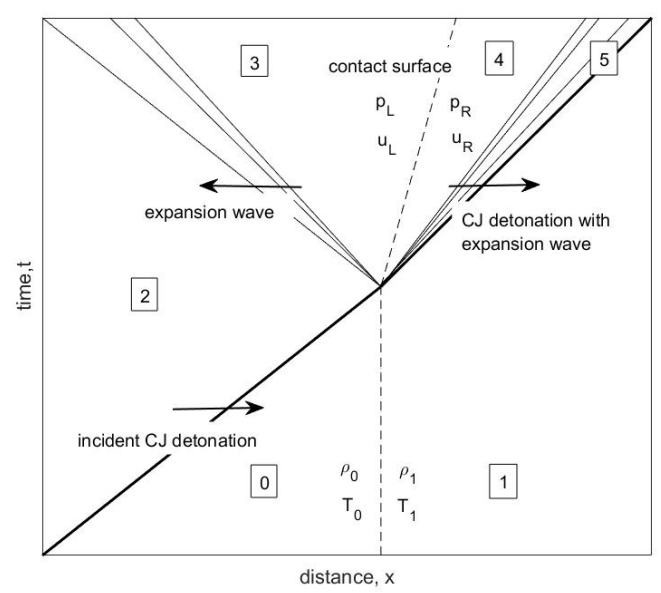

Figure A.4: Case 4: A transmitted CJ detonation with an expansion wave and a reflected expansion wave

$$
\begin{aligned}
& \frac{c_{1}}{\sqrt{\eta_{D, t}}}\left[1-\left(\frac{\gamma+\eta_{D, t}-s}{\gamma+1}\right)\right] \\
& =u_{2}+\frac{2 c_{2}}{\gamma-1}\left[1-\left(\frac{\eta_{D, t}+\gamma(s+1)}{P_{2} \eta_{D, t}(\gamma+1)}\right)^{\frac{\gamma-1}{2 \gamma}}\right]
\end{aligned}
$$

This can be solved iteratively.

\section{Case 4:}

Case 4 is a transmitted CJ detonation with an expansion wave and a reflected expansion wave. It is shown in figure A.4. States 0,1 and 2 are known. Additionally, state 5 can be found using equation 2.1 with $s=0$.

The two sets of expansion wave equations (equation 2.4 and 2.2) are used to relate states 2 and 3 and states 4 and 5 respectively:

$$
u_{3}=u_{2}+\frac{2 c_{2}}{\gamma-1}\left[1-\left(\frac{p_{3}}{p_{2}}\right)^{\frac{\gamma-1}{2 \gamma}}\right]
$$




$$
u_{4}=u_{5}-\frac{2 c_{5}}{\gamma-1}\left[1-\left(\frac{p_{4}}{p_{5}}\right)^{\frac{\gamma-1}{2 \gamma}}\right]
$$

Equating $p$ and $u$ across the contact surface, such that $p_{3}=p_{4}$ and $u_{3}=u_{4}$ gives an equation for $p_{3}$ :

$$
p_{3}^{\frac{\gamma-1}{2 \gamma}}\left[c_{2} p_{2}^{\left(\frac{1-\gamma}{2 \gamma}\right)}+c_{5} p_{5}^{\left(\frac{1-\gamma}{2 \gamma}\right)}\right]=\left(c_{2}+c_{5}\right)-\frac{\gamma-1}{2}\left(u_{5}-u_{2}\right)
$$

which can be solved and then used to find other properties. 


\section{Appendix B}

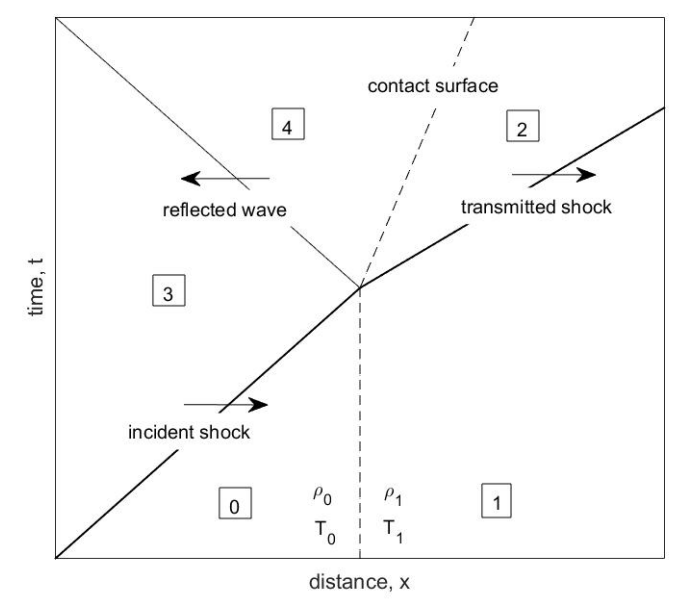

Figure B.1: Transmission of a shock wave across an interface

The transmission of a shock wave across an interface is illustrated in figure B.1. For given incident shock Mach number $M_{s, i}$, the states 0,1 and 3 are known.

State 2 can be found in terms of state 1 and transmitted shock Mach number $M_{s, t}$ using the normal shock equations 2.3:

$$
\begin{aligned}
& \frac{p_{2}}{p_{1}}=\frac{2 \gamma M_{s, t}^{2}-(\gamma-1)}{\gamma+1} \\
& u_{2}=\frac{2 c_{1}}{\gamma+1}\left(M_{s, t}-\frac{1}{M_{s, t}}\right)
\end{aligned}
$$




\section{Density decrease:}

For a density decrease across the interface the reflected wave is an expansion wave. Thus, using equation 2.4 for state 4 :

$$
u_{4}=u_{3}-\frac{2 c_{3}}{\gamma-1}\left[\left(\frac{p_{4}}{p_{3}}\right)^{\frac{\gamma-1}{2 \gamma}}-1\right]
$$

There must be a constant particle velocity across the interface, so $u_{4}=u_{2}$. This gives an equation for $M_{s, t}$ only which can be solved iteratively:

$$
c_{1} \frac{2}{\gamma+1}\left(M_{s, t}-\frac{1}{M_{s, t}}\right)=u_{3}-\frac{2 c_{3}}{\gamma-1}\left[\left(\frac{p_{4}}{p_{3}}\right)^{\frac{\gamma-1}{2 \gamma}}-1\right]
$$

This equation is solved numerically for the specific initial condition of section 4.1.1 to find $M_{s, t}=5.877$, which is then substituted into equation B.1 to find $\frac{p_{2}}{p_{1}}=35.14$.

\section{Density increase:}

For a density increase across the interface, the reflected wave is a shock wave. Thus for state 4:

$$
\begin{aligned}
& \frac{p_{4}}{p_{3}}=\frac{2 \gamma M_{s r}^{2}-(\gamma-1)}{\gamma+1} \\
& u_{4}=\frac{2 c_{3}}{\gamma+1}\left(M_{s, r}-\frac{1}{M_{s, r}}\right)
\end{aligned}
$$

There must be a constant pressure and particle velocity across the interface, so $p_{4}=$ $p_{2}$ and $u_{4}=u_{2}$. This gives two equations with two unknowns $\left(M_{s, t}\right.$ and $\left.M_{s, r}\right)$ which can be simultaneously solved iteratively: 


$$
\begin{aligned}
& p_{1}\left(2 \gamma M_{s, t}^{2}-(\gamma-1)\right)=p_{3}\left(2 \gamma M_{s, r}^{2}-(\gamma-1)\right) \\
& c_{1}\left(M_{s, t}-\frac{1}{M_{s, t}}\right)=c_{3}\left(M_{s, r}-\frac{1}{M_{s, r}}\right)
\end{aligned}
$$

This equation is solved numerically for the specific initial condition of section 4.2.1 to find $M_{s, t}=6.832$, which is then substituted into equation B.1 to find $\frac{p_{2}}{p_{1}}=46.61$. 


\section{References}

[1] R.A. Strehlow, R.J. Stiles, and A.A. Adamczyk. Transient studies of detonation waves. Astronautica Acta, 17(4-5):509, 1972.

[2] D. Bjerketvedt, O.K. Sonju, and I.O. Moen. The influence of experimental condition on the reinitiation of detonation across an inert region. Progress in Astronautics and Aeronautics, 106:109-130, 1986.

[3] G.O. Thomas, P. Sutton, and D.H. Edwards. The behavior of detonation waves at concentration gradients. Combustion and flame, 84(3-4):312-322, 1991.

[4] D.H. Lieberman and J.E. Shepherd. Detonation interaction with an interface. Physics of Fluids, 19(9):096101, 2007.

[5] S. Boulal, P. Vidal, and R. Zitoun. Experimental investigation of detonation quenching in non-uniform compositions. Combustion and Flame, 172:222-233, 2016.

[6] M.S. Kuznetsov, V.I. Alekseev, S.B. Dorofeev, I.D. Matsukov, and J.L. Boccio. Detonation propagation, decay, and reinitiation in nonuniform gaseous mixtures. Symposium (International) on Combustion, 27(2):2241-2247, 1998.

[7] M.S. Kuznetsov, S.B. Dorofeev, A.A. Efimenko, V.I. Alekseev, and W. Breitung. Experimental and numerical studies on transmission of gaseous detonation to a less sensitive mixture. Shock Waves, 7(5):297-304, 1997. 
[8] J. Li, W.H. Lai, K. Chung, and F.K. Lu. Experimental study on transmission of an overdriven detonation wave from propane/oxygen to propane/air. Combustion and Flame, 154(3):331-345, 2008.

[9] H.D. Ng, B. Botros, J. Chao, J. Yang, N. Nikiforakis, and J.H.S. Lee. Head-on collision of a detonation with a planar shock wave. Shock Waves, 15(5):341-352, 2006.

[10] B.B. Botros, H.D. Ng, Y.J. Zhu, Y. Ju, and J.H.S. Lee. The evolution and cellular structure of a detonation subsequent to the head-on interaction with a shock wave. Combustion and Flame, 151:573-580, 2007.

[11] J.H.S. Lee. The Detonation Phenomenon. Cambridge University Press, Cambridge; New York, 2008.

[12] A.J. Higgins. In F. Zhang, Shock Waves Science and Technology Library, volume 6, pages 33-105. Springer, 2012.

[13] Y.B. Zeldovich. On the theory of the propagation of detonation in gaseous systems. 1950.

[14] J. von Neumann. Theory of detonation waves. Technical report OSRD-549. Technical Report OSRD-549, National Defense Research Committee, 1942.

[15] W. Döring. Über den detonationsvorgang in gasen. Annalen der Physik, 435(67):421-436, 1943.

[16] W.J.M. Rankine. On the thermodynamic theory of waves of finite longitudinal disturbance. Philosophical Transactions of the Royal Society of London, pages 277-288, 1870. 
[17] H. Hugoniot. Mémoire sur la propagation du mouvement dans les corps et spécialement dans les gaz parfaits. Gauthier-Villars, 1887.

[18] B. Riemann. Über die Fortpflanzung ebener Luftwellen von endlicher Schwingungsweite. Verlag der Dieterichschen Buchhandlung, 1860.

[19] S. Arrhenius. Über die dissociationswärme und den einfluss der temperatur auf den dissociationsgrad der elektrolyte. Zeitschrift für physikalische Chemie, 4(1):96-116, 1889.

[20] M. Short and G.J. Sharpe. Pulsating instability of detonations with a two-step chainbranching reaction model: theory and numerics. Combustion Theory and Modelling, $7(2): 401-416,2003$.

[21] E.F. Toro. Riemann Solvers and Numerical Methods for Fluid Dynamics : A Practical Introduction. Springer, Berlin, 2006.

[22] H.D. Ng, N. Nikiforakis, and J.H.S. Lee. Assessment of a high resolution centered scheme for detonation modelling. Technical report, 2000.

[23] H.D. Ng, M.I. Radulescu, A.J. Higgins, N. Nikiforakis, and J.H.S. Lee. Numerical investigation of the instability for one-dimensional chapman-jouguet detonations with chain-branching kinetics. Combustion Theory and Modelling, 9(3):385-401, 2005.

[24] A. Haselbacher. On impedance in shock-refraction problems. Shock Waves, $22(4): 381-384,2012$.

[25] I.I. Glass and J.P. Sislian. Nonstationary flows and shock waves, volume 39. Oxford University Press on Demand, 1994. 